Draft VERSion OCTOBER 22, 2018

Preprint typeset using IATEX style emulateapj v. 2/16/10

\title{
EDDINGTON-LIMITED ACCRETION AND THE BLACK HOLE MASS FUNCTION AT REDSHIFT 6
}

\author{
Chris J. Willott ${ }^{1}$, Loic Albert ${ }^{2}$, Doris Arzoumanian ${ }^{3}$, Jacqueline Bergeron ${ }^{4}$, David Crampton ${ }^{1}$, Philippe \\ Delorme $^{5}$, John B. Hutchings ${ }^{1}$, Alain Omont ${ }^{4}$, Céline Reylé ${ }^{6}$, and David Schade ${ }^{1}$, \\ Draft version October 22, 2018
}

\begin{abstract}
We present discovery observations of a quasar in the Canada-France High- $z$ Quasar Survey (CFHQS) at redshift $z=6.44$. We also use near-IR spectroscopy of nine CFHQS quasars at $z \sim 6$ to determine black hole masses. These are compared with similar estimates for more luminous Sloan Digital Sky Survey (SDSS) quasars to investigate the relationship between black hole mass and quasar luminosity. We find a strong correlation between Mg II FWHM and UV luminosity and that most quasars at this early epoch are accreting close to the Eddington limit. Thus these quasars appear to be in an early stage of their life cycle where they are building up their black hole mass exponentially. Combining these results with the quasar luminosity function, we derive the black hole mass function at $z=6$. Our black hole mass function is $\sim 10^{4}$ times lower than at $z=0$ and substantially below estimates from previous studies. The main uncertainties which could increase the black hole mass function are a larger population of obscured quasars at high-redshift than is observed at low-redshift and/or a low quasar duty cycle at $z=6$. In comparison, the global stellar mass function is only $\sim 10^{2}$ times lower at $z=6$ than at $z=0$. The difference between the black hole and stellar mass function evolution is due to either rapid early star formation which is not limited by radiation pressure as is the case for black hole growth or inefficient black hole seeding. Our work predicts that the black hole mass - stellar mass relation for a volume-limited sample of galaxies declines rapidly at very high redshift. This is in contrast to the observed increase at $4<z<6$ from the local relation if one just studies the most massive black holes.

Subject headings: cosmology: observations — quasars: general — quasars: emission lines
\end{abstract}

\section{INTRODUCTION}

Quasars are very luminous objects residing at the centers of galaxies believed to be powered by accretion of matter onto a supermassive black hole. The high luminosity makes them identifiable out to very high redshift where they can be used to study black hole growth, galaxy evolution and the intergalactic medium. It has been discovered that most nearby, massive galaxies harbor central supermassive black holes and that the mass of the black hole scales with galaxy properties such as bulge luminosity (Magorrian et al. 1998) and velocity dispersion (Ferrarese \& Merritt 2000; Gebhardt et al. 2000). These observations suggest that black holes play an important role in galaxy evolution, likely due to feedback which heats and expels gas which would otherwise form stars in the galaxy (Silk \& Rees 1998; Di Matteo et al. 2005, Hopkins et al. 2006a; Croton et al. 2006). The physical details of how the energy emitted by the active galactic nucleus (AGN) couples to the gas are still poorly understood.

The relic black hole mass function at low redshift is the

\footnotetext{
${ }^{1}$ Herzberg Institute of Astrophysics, National Research Council, 5071 West Saanich Rd, Victoria, BC V9E 2E7, Canada; chris.willott@nrc.ca

${ }^{2}$ Canada-France-Hawaii Telescope Corporation, 65-1238 Mamalahoa Highway, Kamuela, HI96743, USA

${ }^{3}$ CEA-Saclay, IRFU, SAp, 91191, Gif-sur-Yvette, France

${ }^{4}$ Institut d'Astrophysique de Paris, CNRS and Université Pierre et Marie Curie, 98bis Boulevard Arago, F-75014, Paris, France

${ }^{5}$ School of Physics \& Astronomy, University of St Andrews, North Haugh, St Andrews KY16 9SS, UK

${ }^{6}$ Institut Utinam, Observatoire de Besançon, Université de Franche-Comté, BP1615, 25010 Besançon Cedex, France
}

result of all the black hole growth via accretion and merging over cosmic time. There is good agreement between the number density of black holes at low redshift and the observed accretion as inferred by studies of AGN luminosity functions if black holes accrete with a mass-toenergy conversion efficiency in the range $0.06 \lesssim \epsilon \lesssim 0.10$ (Yu \& Tremaine 2002; Marconi et al. 2004; Hopkins et al. 2007; Shankar et al. 2009). Such studies also agree with luminosity function observations at various redshifts (e.g. Ueda et al. 2003) that there is downsizing in the AGN population, that the more massive black holes were built up more rapidly at high-redshift than the less massive black holes which accreted a greater fraction of their mass at lower redshift.

Reverberation mapping of broad-lined AGNs at lowredshift has shown that a relationship exists between the distance of the line-emitting gas from the central ionizing sources, $R$, and the optical/UV luminosity, $L$ (Kaspi et al. 2000; Bentz et al. 2009). This has the form $R \propto$ $L^{0.5}$, as expected based on simple ionization models. The consequence of this relationship is that measurement of the velocity of the line-emitting gas, e.g. via its Dopplerbroadened linewidth, and the luminosity are sufficient to determine the gravitational mass of the central black hole (Wandel et al. 1999). Such measurements can be made with single epoch, moderate signal-to-noise $(\mathrm{S} / \mathrm{N})$ spectra out to very high redshifts. This method appears to give a fairly low scatter (between 0.2 to 0.3 dex for the $\mathrm{H} \beta$ and $\mathrm{Mg}$ II lines; Kollmeier et al. 2006; Fine et al. 2008; Shen et al. 2008; Steinhardt \& Elvis 2010), comparable with that of reverberation mapping. For a review of the reliability and accuracy of this method we refer the reader to Peterson (2010). 
Using this method it has been possible to measure black hole masses for SDSS quasars at $z=6$ (Willott et al. 2003; Jiang et al. 2007; Kurk et al. 2007; Kurk et al. 2009). Most of these are from the main SDSS sample of Fan et al. (2006) which contains very luminous quasars. These results show that the most luminous $z=6$ quasars contain black hole masses $M_{\mathrm{BH}}>10^{9} M_{\odot}$ accreting at close to the Eddington limit. This is not too surprising because if they were to accrete at substantially below the Eddington limit, the required black hole masses would be $M_{\mathrm{BH}}>10^{10} M_{\odot}$ and such black holes are rare even at more moderate redshifts (McLure \& Dunlop 2004; Vestergaard \& Osmer 2009). If there are sub-Eddington accreting black holes at $z=6$ they would be found in the lower luminosity quasar samples such as the SDSS deep stripe (Jiang et al. 2009) and Canada-France High-z Quasar Survey (CFHQS; Willott et al. 2010). Two of the SDSS deep stripe quasars have black hole mass measurements and both are found to have lower black hole masses than the SDSS main quasars and are accreting at approximately the Eddington limit (Kurk et al. 2007; 2009). In order to increase the numbers of low luminosity quasars with black hole mass measurements and determine if any of them are powered by high mass black holes accreting at low Eddington ratios, we are carrying out a near-IR spectroscopy program on all $z>6$ CFHQS quasars.

The existence of $M_{\mathrm{BH}}>10^{9} M_{\odot}$ black holes at redshift 6 has led to much theoretical work to explain how such objects can be built up in the $<1$ Gyr of cosmic time available. Standard Eddington-limited accretion has an e-folding time (the Salpeter time-scale) of $4.5 \times 10^{7} \mathrm{yr}$ assuming efficiency $\epsilon=0.1$. This led some people to propose that episodes of super-Eddington accretion at very high redshift were necessary (Volonteri \& Rees 2005; Kawakatu \& Wada 2009). Another possibility is higher efficiency which lowers the Salpeter time-scale and may be expected for rapidly spinning black holes (Shapiro 2005). Other studies have shown that Eddington-limited accretion can account for these black holes, but only if very massive seed black holes or multiple stellar seeds are invoked (Yoo \& Miralda-Escudé 2004; Sijacki et al. 2009). Mergers are also an important part of the growth of the most massive black holes at high redshift (Volonteri \& Rees 2006; Li et al. 2007).

An important prediction of galaxy evolution models is the evolution of the black hole mass - stellar mass relation. There have been many attempts at observational determinations of this ratio, however selection effects are critical. Studies involving AGN tend to show higher than local ratios of $M_{\mathrm{BH}} / M_{\text {stellar }}$ at all redshifts; $z<1$ (Mathur \& Grupe 2005; Woo et al. 2008), $1<z<4$ (Peng et al. 2006; McLure et al. 2006; Merloni et al. 2010); $z>4$ (Walter et al. 2004; Riechers et al. 2008; Wang et al. 2010), whereas studies based on $z \approx 2$ starburst galaxies show lower ratios (Borys et al. 2005; Alexander et al. 2008). Other approaches consider the global evolution of the stellar mass function and black hole mass function using various constraints such as the observed quasar luminosity function, the relic black hole mass function, the X-ray background or results from theoretical simulations (Hopkins et al. 2006b; Di Matteo et al. 2008; Somerville 2009; Shankar et al. 2009). Such studies have shown fairly little evolution in this ratio, at
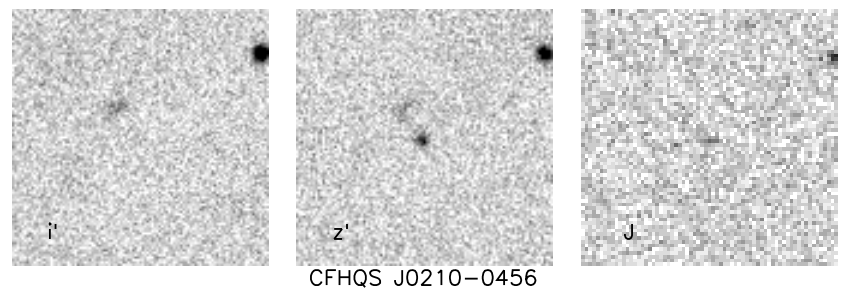

Figure 1. Images in the $i^{\prime}, z^{\prime}$ and $J$ filters centered on CFHQS J0210-0456. Each image covers $20^{\prime \prime} \times 20^{\prime \prime}$. The images are oriented with north up and east to the left.

least out to $z=2$ where they are most strongly constrained by data.

In this paper we present new data on CFHQS quasars designed to address the growth of black holes at early times. In section 2 we present the discovery of the most distant known quasar at $z=6.44$. Near-IR spectroscopy of nine CFHQS quasars is presented in Section 3. In Section 4 we use these data to derive black hole masses, investigate correlations between FWHM, luminosity and black hole mass and determine the Eddington ratio distribution and its implications. Section 5 combines this work with the $z=6$ quasar luminosity function of Willott et al. (2010) to determine the black hole mass function and compare its evolution with that of the global stellar mass function. In the appendix we show data on four quasars for which no black hole mass measurement was possible and discuss why.

All optical and near-IR magnitudes in this paper are on the $\mathrm{AB}$ system. Cosmological parameters of $H_{0}=$ $70 \mathrm{~km} \mathrm{~s}^{-1} \mathrm{Mpc}^{-1}, \Omega_{\mathrm{M}}=0.28$ and $\Omega_{\Lambda}=0.72$ (Komatsu et al. 2009) are assumed throughout.

\section{DISCOVERY OF A NEW CFHQS QUASAR AT $Z=6.44$}

In previous papers, we have presented imaging and optical spectroscopy of 19 CFHQS quasars. Since these publications, we have discovered one more CFHQS

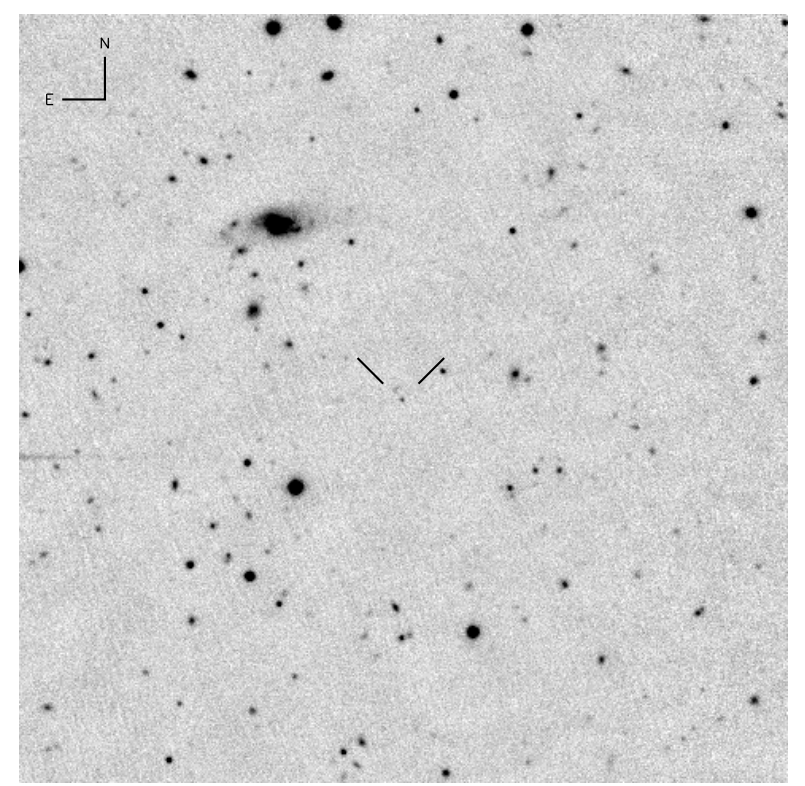

Figure 2. $\quad z^{\prime}$-band finding chart for CFHQS J0210-0456. The field of view is $3^{\prime} \times 3^{\prime}$. 
Table 1

New quasar position and photometry

\begin{tabular}{|c|c|c|c|c|c|c|c|}
\hline Quasar & "RA and DEC (J2000.0) & $\overline{i^{\prime} \mathrm{mag}}$ & 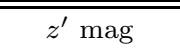 & $\overline{J \text { mag }}$ & $\overline{i^{\prime}-z^{\prime}}$ & $\overline{z z^{\prime}-J}$ & $M_{1450}$ \\
\hline FHQS J021013-045620 & $02: 10: 13.19-04: 56: 20.9$ & $>25.74^{\mathrm{a}}$ & $22.67 \pm 0.05$ & $22.28 \pm 0.27$ & $>3.07$ & $0.39 \pm 0.28$ & -24.28 \\
\hline
\end{tabular}

Notes. All magnitudes are on the AB system.

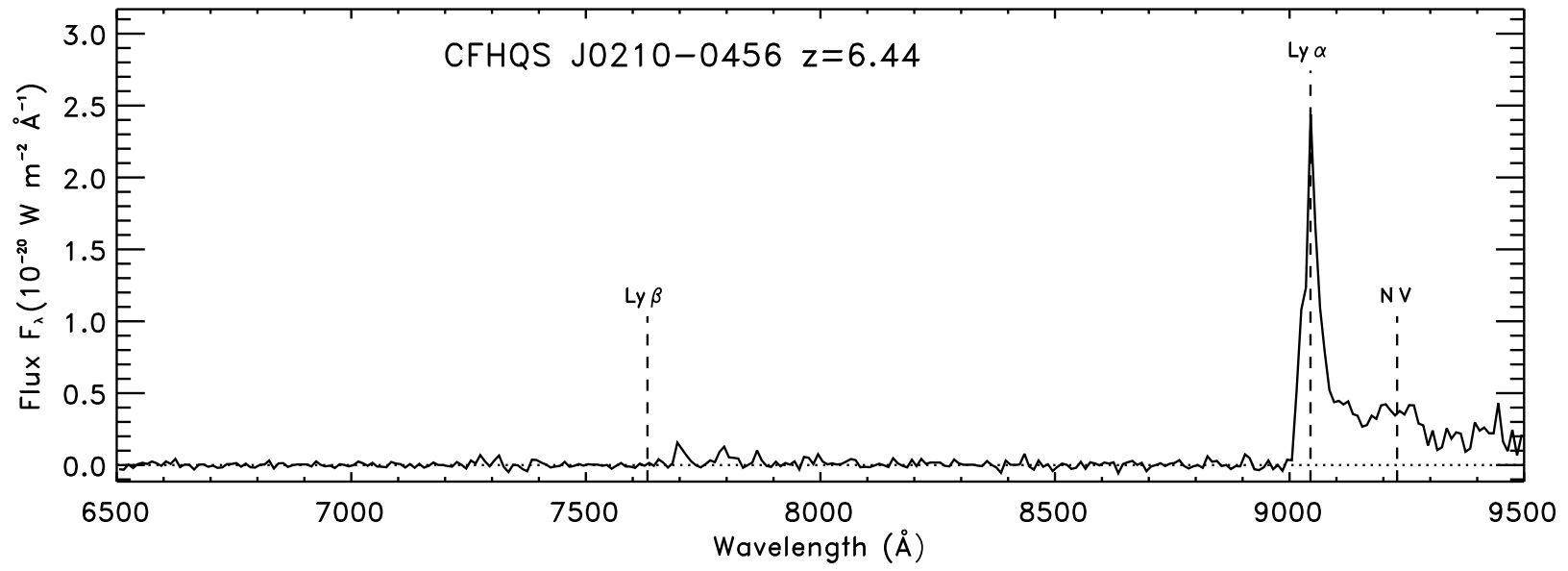

Figure 3. Optical spectrum of the newly discovered quasar. The locations of Ly $\alpha$, Ly $\beta$ and $\mathrm{N} v$ for a redshift of $z=6.44$ are marked with dashed lines. The spectrum is binned in $10 \AA$ pixels.

quasar. In this section we present the observations and features of this quasar. The quasar was discovered by its red $i^{\prime}-z^{\prime}$ color in imaging in the CFHT Legacy Survey Wide W1 field7 . Follow-up near-IR imaging observations at the $3.6 \mathrm{~m}$ ESO New Technology Telescope with the SOFI instrument showed that it has a relatively blue $z^{\prime}-J$ color and is therefore more likely to be a highredshift quasar than a $\mathrm{T}$ dwarf (see Figure 1 of Willott et al. 2009). Figure 1 shows small regions centred on this object in the $i^{\prime}, z^{\prime}$ and $J$ filters. The object is not detected at $i^{\prime}$ and only weakly detected at $J$. A larger $z^{\prime}$-band field is shown in Figure 2 which can be used as a finding chart. Photometry and the position of this quasar, named CFHQS J021013-045620, are given in Table 1 (note that from here on we use the abbreviated version of the name).

Spectroscopy of the quasar was obtained on 2009 November 12 and 2009 December 17 with the GMOS spectrograph at the Gemini-South telescope. The R400 grating was used with a 1 arcsec slit to give a resolving power of 1300 . The seeing was 0.8 arcsec and the sky transparency was photometric. The total integration time was 3.5 hours. The nod-and-shuffle mode was employed to enable very accurate sky subtraction. The data reduction method is as described in Willott et al. (2007).

Figure 3 presents the reduced optical spectrum of CFHQS J0210-0456. The most obvious feature in the spectrum is the strong, narrow, asymmetric Ly $\alpha$ emission line. The peak of this emission line is at $9045 \AA$

\footnotetext{
${ }^{8}$ http://www.cfht.hawaii.edu/Science/CFHTLS
}

which is equal to a redshift of $z=6.44$. Accounting for some absorption on the blue wing of the line, the estimated intrinsic FWHM of the line is $1300 \mathrm{~km} \mathrm{~s}^{-1}$. The redshift of the peak does not change appreciably when making such an absorption correction (peak shifts to $9040 \AA$ ). This spectrum shows several other characteristics of $z>6$ quasars. There is a sharp break in the continuum level across the Ly $\alpha$ line due to foreground neutral hydrogen absorption. The continuum flux at observed-frame $7800 \AA$ is clearly visible which corresponds to light allowed to pass through the IGM at $z \approx 5$.4. Shortward of the expected location of $\mathrm{Ly} \beta$ there is complete absorption again. There is a possible $\mathrm{N} \mathrm{v}$ broad emission line which is marked on Figure 3.

In the following section we will describe near-IR spectroscopy of a sample of CFHQS quasars. One of the quasars observed is CFHQS J0210-0456. Although this quasar is extremely faint in the near-IR, a 4 hours integration with NIRI on Gemini-North allowed the detection of the $\mathrm{Mg}$ II $\lambda 2799$ emission line (Figure 4). The best-fit Mg II redshift is $z=6.438 \pm 0.004$. This is statistically identical to the redshift of the peak of Ly $\alpha$. The closeness of the $\operatorname{Ly} \alpha$ peak and Mg II redshifts has been noted before for many CFHQS quasars (Willott et al. 2010) and will be investigated in a future paper. This redshift of $z=6.438$ is the highest of any known quasar, just surpassing SDSS J1148+5251 at $z=6.419$ (Fan et al. 2003; Walter et al. 2003) and CFHQS J2329-0301 at $z=6.417$ (Section 3).

The accurate redshift for CFHQS J0210-0456 allows us to measure the size of the ionized region in front of the quasar via the physical extent of measurable flux on the 
blue side of Ly $\alpha$. This can give important information on the ionization state of the IGM surrounding the quasar (Cen \& Haiman 2000; Wyithe \& Loeb 2004; Bolton \& Haehnelt 2007). The spectrum of CFHQS J0210-0456 reaches zero flux at $9005 \AA$, which corresponds to a ionized near-zone size of just 1.7 proper Mpc. Note that using the wavelength at which the transmission reaches $10 \%$, as used by Fan et al. (2006), rather than where the flux reaches zero, gives almost exactly the same size due to the steepness of the spectrum. Given this small size and steep drop, we do not bin the spectrum in $20 \AA$ bins (as Fan et al. did) since then the measured size would be very dependent upon the choice of bins.

This size of $1.7 \mathrm{Mpc}$ is extremely small, much smaller than any other known $z=6$ quasars with the exception of lineless or BAL quasars and less than the typical size for SDSS quasars at $z>6.1$ of $5 \mathrm{Mpc}$ (Carilli et al. 2010). Note however that if these near-zone sizes are set by the expansion of an ionization front from the quasar then they are expected to scale with the quasar luminosity as $R \propto L^{1 / 3}$. Since CFHQS J0210-0456 has a luminosity a factor of ten lower than SDSS quasars, then the luminosity-scaled near-zone size is consistent with that expected based on the near-zone sizes of the SDSS $z>6.1$ quasars. Further exploration of the near-zone size and other constraints on the ionization state of the IGM using this quasar will be presented elsewhere.

\section{NEAR-INFRARED SPECTROSCOPY OBSERVATIONS}

We are attempting $K$-band spectroscopy of all CFHQS quasars with redshifts $z>6$. We do not target the $z<6$ quasars because the Mg II line center lies at wavelengths $<1.96 \mu \mathrm{m}$ and there is substantial atmospheric absorption shortward of this wavelength which could affect determining a reliable continuum and line width. Of the 20 quasars found in the CFHQS so far, 5 were not targeted because they had $z<6$. Two $z>6$ quasars have not yet been targeted due to their recent discovery. Therefore a total of 13 quasars have had near-IR spectroscopy attempted.

One quasar, CFHQS J1509-1749, already has a published near-IR spectrum from the GNIRS instrument at Gemini-South (Willott et al. 2007). Near-infrared spectroscopy of the other 12 quasars was obtained using the NIRI instrument on the Gemini-North telescope. The $K$ band grism covers the wavelength range $1.87-2.59 \mu \mathrm{m}$ at a resolution of $R=520\left(570 \mathrm{~km} \mathrm{~s}^{-1}\right)$ with the 0.75 arcsec slit. The seeing was generally good - in the range 0.5 to 0.7 arcsec. The observations of each target were split into 300 second frames nodded along the slit to enable good sky subtraction and bad pixel rejection. All frames were checked for the fixed pattern noise problem that randomly affects some NIRI exposures. Any pattern noise affected frames or frames taken in variable sky conditions were discarded. Total usable integration times ranged from 1.3 to 4 hours with an average of 2.8 hours.

Data reduction followed standard near-IR longslit procedures which will be briefly described here. Flat-field frames obtained from continuum lamp exposures were combined to make a flat-field image used to flatten the individual 300 second frames. First-pass sky subtraction was performed by determining the $2 \mathrm{D}$ sky spectrum from neighboring frames obtained within a time-span of \pm 450 seconds. Residual sky emission was removed by fitting a spline along the column of each spectral row. The spatial shifts between the individual frames were determined via measurement of the quasar location to enable all the frames to be combined into a single $2 \mathrm{D}$ spectrum. The combination process averaged the pixel fluxes using positive and negative sigma clipping and bad pixel mask rejection. Wavelength calibration was performed using a 4th order polynomial fit to an argon lamp spectrum. The residuals of this fit about the known wavelengths were around $0.7 \AA$. Relative flux calibration and atmospheric absorption corrections were made using spectra of early A-type stars observed at similar airmass. Absolute flux calibration was performed using broad band photometry. The uncertainty as a function of wavelength was determined by measuring an iterative sigma-clipped rms along the clean parts of each column (spatial direction) in the $2 \mathrm{D}$ spectrum.

Fig 4 shows the resulting spectra of 9 of the 13 observed quasars and the $1 \sigma$ uncertainty. The $\mathrm{S} / \mathrm{N}$ per pixel in the continuum at clean parts of the spectra ranges from 3 to 20 depending upon the source flux-density, total integration time and observing conditions. For the other 4 quasars the spectra are not suitable for the measurement of the $\mathrm{Mg}$ II line due to insufficient $\mathrm{S} / \mathrm{N}$. These observations are discussed in the appendix. As explained there, we do not believe the exclusion of these quasars from the following analysis causes any biases. Although at first sight there appear to be Mg II associated absorption lines for some quasars, none are detected at high significance. Most apparent absorption lines are at wavelengths of night sky emission lines where the noise is higher. These will not strongly affect the fitted Mg II emission lines because such data points have low weight in the fit.

The spectra consist of 3 possible components: a power law continuum, a Doppler-broadened blend of many iron emission lines and the Mg II $2799 \AA$ emission line doublet. A simultaneous fit to these three components was carried out. The iron line emission template is from McLure \& Dunlop (2004) and covers the spectral range $2100-$ $3085 \AA$. Therefore only data in this rest-frame wavelength range was included in the fit. There are six free parameters in the fit: (i) the continuum normalization; (ii) the continuum power law slope; (iii) the redshift of the iron and Mg II broad emission lines (assumed equal); (iv) the normalization of the iron template; (v) the peak height of the Gaussian Mg II emission line; (vi) the FWHM of the $\mathrm{Mg}$ II doublet.

The best-fit was determined by minimizing the $\chi^{2}$ using an amoeba algorithm (Press et al. 1992). The bestfit continuum and total spectra are plotted on top of the data in Figure 4. In all cases, the fits provide a good description of the data. Bootstrap resampling was used to derive uncertainties on the fitted parameters. 500 randomly-perturbed realizations of each spectrum were generated and fit by $\chi^{2}$ minimization.

Table 2 presents the best-fit Mg II redshift, FWHM velocities and their uncertainties for the nine CFHQS quasars. The broad line FWHM values in this table were determined by deconvolving the instrumental resolution $\left(570 \mathrm{~km} \mathrm{~s}^{-1}\right.$ for the NIRI spectra and $330 \mathrm{~km} \mathrm{~s}^{-1}$ for the GNIRS spectrum). Note that in all cases the deconvolved 

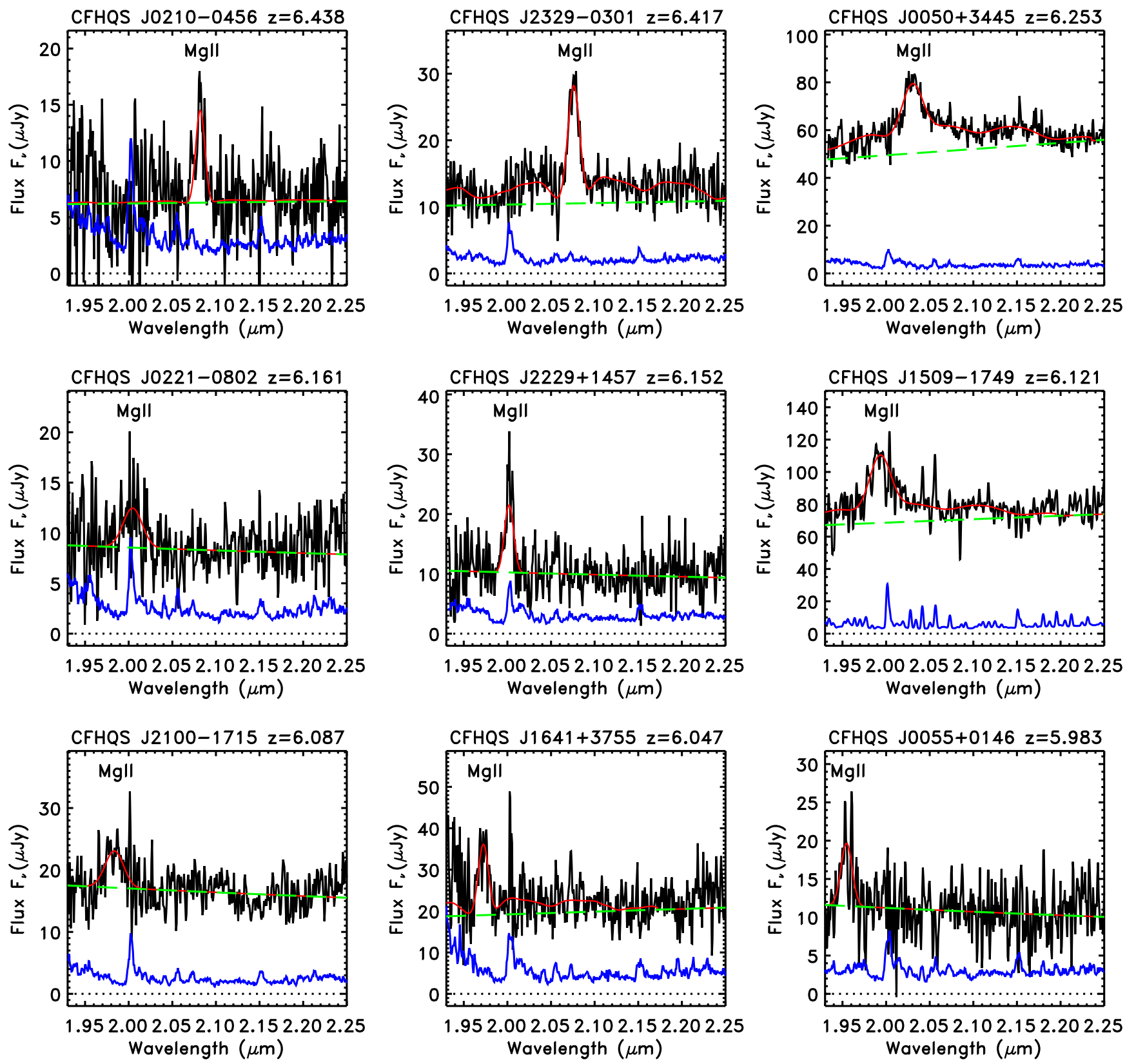

Figure 4. $K$-band spectra of CFHQS quasars (black line). The fitted model of power law continuum, broadened Fe template and broadened Mg II doublet is shown as a red line. The power law continuum only is shown as a green dashed line. At the bottom of the plot, the blue line is the $1 \sigma$ noise spectrum. The location of the Mg II broad emission line is labeled. Note how narrow most of the Mg II lines are.

FWHM is greater than twice the instrumental FWHM meaning that the observations were well-resolved. The redshift uncertainty includes 0.002 added in quadrature due to the absolute wavelength calibration uncertainty of the spectroscopy. It does not include any extra uncertainty due to the fact the line shape may not be a perfect gaussian due to asymmetric emission or absorption lines.

\section{BLACK HOLE MASSES IN $Z \sim 6$ QUASARS}

\subsection{Black hole mass determinations}

The successful measurement of the Mg II emission line widths in these quasars allows us to determine black hole masses by the virial method. As described in Section
1, despite several potential sources of uncertainty, this method appears to give black hole masses accurate to $\approx 0.3 \mathrm{dex}$ (Shen et al. 2008; Steinhardt \& Elvis 2010) if high quality line and continuum measurements are available. We use the relationship determined by Vestergaard \& Osmer (2009) to calculate black hole masses from the Mg II line width, FWHM (Mg II) and the luminosity at rest-frame $3000 \AA, L_{3000}$

$$
\log M_{\mathrm{BH}}=6.86+2 \log \frac{\mathrm{FWHM}(\mathrm{Mg} \mathrm{II})}{1000 \mathrm{~km} \mathrm{~s}^{-1}}+0.5 \log \frac{L_{3000}}{10^{37} \mathrm{~W}} .
$$

Values of $L_{3000}$ are derived from the fitted continuum normalization and their uncertainties include $10 \%$ added 
Table 2

Measured and derived parameters of CFHQS quasars

\begin{tabular}{ccccccc}
\hline Quasar & $\begin{array}{c}\text { Mg II redshift } \\
z_{\mathrm{Mg} \mathrm{II}}\end{array}$ & $M_{1450}$ & $\begin{array}{c}\text { FWHM (Mg II }) \\
\left(\mathrm{km} \mathrm{s}^{-1}\right)\end{array}$ & $\begin{array}{c}L_{3000} \\
\left(10^{37} \mathrm{~W}\right)\end{array}$ & $\begin{array}{c}M_{\mathrm{BH}} \\
\left(M_{\odot}\right)\end{array}$ & $\begin{array}{c}\lambda \\
\left(\equiv L_{\mathrm{Bol}} / L_{\mathrm{Edd}}\right)\end{array}$ \\
\hline CFHQS J0210-0456 & $6.438 \pm 0.004$ & -24.28 & $1300 \pm 350$ & $42 \pm 5$ & $\left(8.0_{-4.5}^{+5.5}\right) \times 10^{7}$ \\
CFHQS J2329-0301 & $6.417 \pm 0.002$ & -25.00 & $2020 \pm 110$ & $71 \pm 8$ & $\left(2.5_{-0.4}^{+0.4}\right) \times 10^{8}$ \\
CFHQS J0050+3445 & $6.253 \pm 0.003$ & -26.62 & $4360 \pm 270$ & $342 \pm 34$ & $\left(2.6_{-0.4}^{+0.5}\right) \times 10^{9}$ & 0.6 \\
CFHQS J0221-0802 & $6.161 \pm 0.014$ & -24.45 & $3680 \pm 1500$ & $50 \pm 5$ & $\left(7.0_{-4}^{+7.5}\right) \times 10^{8}$ & 0.33 \\
CFHQS J2229+1457 & $6.152 \pm 0.003$ & -24.52 & $1440 \pm 330$ & $60 \pm 6$ & $\left(1.2_{-0.5}^{+0.7}\right) \times 10^{8}$ & 2.4 \\
CFHQS J1509-1749 & $6.121 \pm 0.002$ & -26.78 & $4420 \pm 130$ & $440 \pm 44$ & $\left(3.0_{-0.3}^{+0.3}\right) \times 10^{9}$ & 0.68 \\
CFHQS J2100-1715 & $6.087 \pm 0.005$ & -25.03 & $3610 \pm 420$ & $98 \pm 10$ & $\left(9.4_{-2.5}^{+2.9}\right) \times 10^{8}$ & 0.49 \\
CFHQS J1641+3755 & $6.047 \pm 0.003$ & -25.19 & $1740 \pm 190$ & $120 \pm 13$ & $\left(2.4_{-0.8}^{+1.0}\right) \times 10^{8}$ & 2.3 \\
CFHQS J0055+0146 & $5.983 \pm 0.004$ & -24.53 & $2040 \pm 280$ & $63 \pm 7$ & $\left(2.4_{-0.7}^{+0.9}\right) \times 10^{8}$ \\
\hline
\end{tabular}

Notes. - Mg II redshifts are based on the wavelength of the fitted Mg II doublet and in all cases supersede previously published values which were based on $\operatorname{Ly} \alpha$ in the optical spectra.
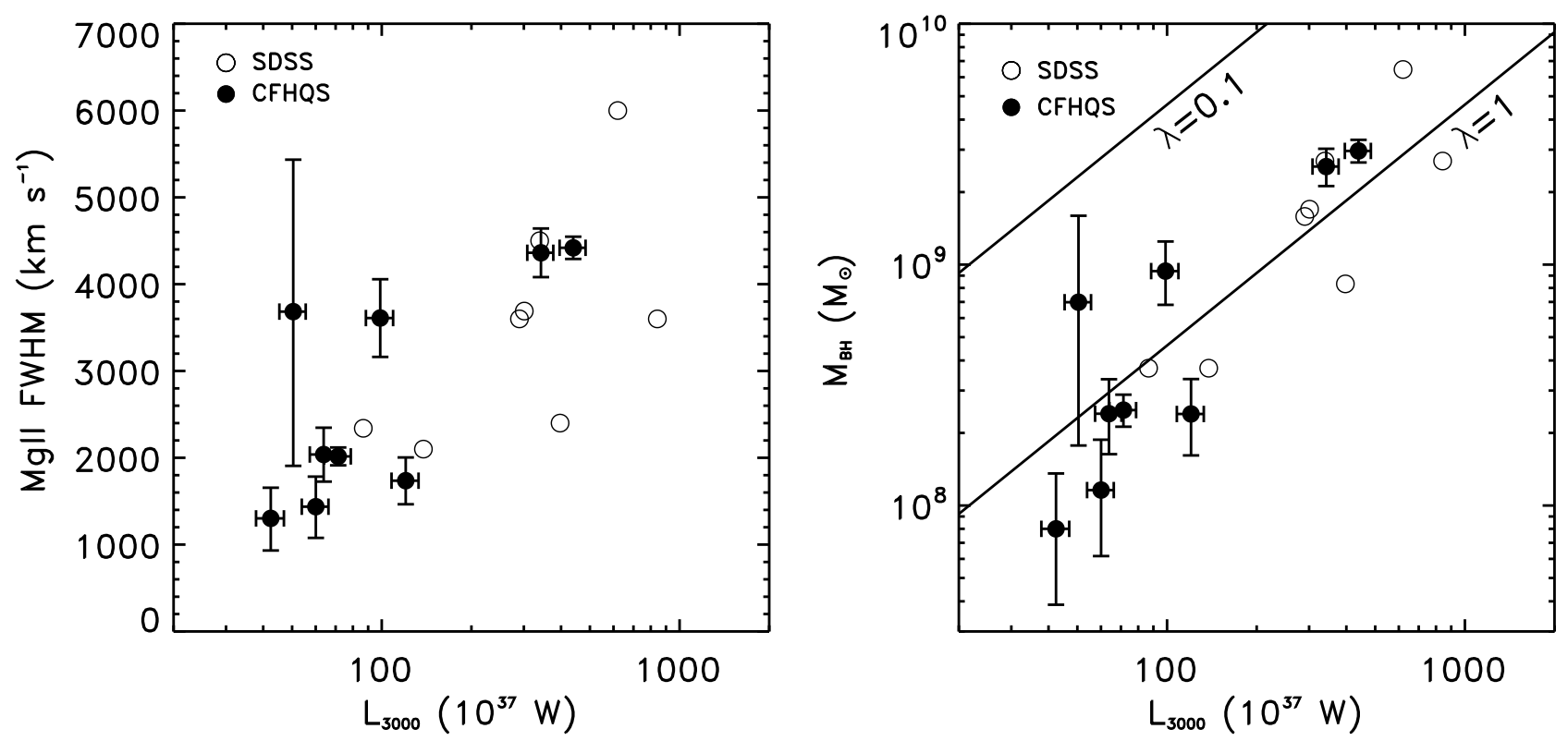

Figure 5. Left: Mg II FWHM against luminosity $L_{3000}$ for CFHQS and SDSS $z \approx 6$ quasars. Error bars are only plotted for the CFHQS quasars. There is a strong positive correlation between the two independently-measured quantities. Right: Virial black hole mass against luminosity for the same quasars. Also plotted are lines corresponding to Eddington ratios of 0.1 and 1. Quasars from both samples are accreting at close to the Eddington luminosity.

in quadrature to account for the absolute flux calibration uncertainty. No additional uncertainty due to variability was included. The black hole mass uncertainties were determined by inserting the extreme values of the luminosity and linewidth, based on their rms uncertainties, in the above equation (rather than adding errors in quadrature). No additional uncertainty was added to account for the fact that the Mg II virial relation for black hole masses has its own scatter of about 0.3 dex. The derived values for $L_{3000}$ and $M_{\mathrm{BH}}$ are given in Table2.

As noted in Section 3, the spectra have differing $\mathrm{S} / \mathrm{N}$ due to the fact that some of these quasars are very faint. Therefore we have to consider whether the quasars with low $\mathrm{S} / \mathrm{N}$ might have their line width measurements biased high or low due to this. Shen et al. (2008) studied virial black hole mass determinations from SDSS DR5 quasar spectroscopy. Their $\mathrm{S} / \mathrm{N}$ distribution is similar to that of the CFHQS spectra. They found that there was no dependence of the FWHM distribution on the
$\mathrm{S} / \mathrm{N}$ of the spectra. Denney et al. (2009) found a systematic shift towards lower measured black hole masses of $\sim 0.05$ dex as the $\mathrm{S} / \mathrm{N}$ per pixel was artificially decreased from 20 to 5 . Given that most of our spectra have $\mathrm{S} / \mathrm{N}$ per pixel $>5$, we do not consider this bias to be important for our study (0.05 dex is substantially less than the $M_{\mathrm{BH}}$ uncertainty due to propagating the measured line width uncertainties for the quasars with low $\mathrm{S} / \mathrm{N})$. .

The CFHQS $z>6$ quasars are mostly of moderate optical luminosity. In order to sample a broad range of luminosity at a fixed redshift we also include in our analysis the published results of near-IR MgII spectroscopy of SDSS $z \approx 6$ quasars. The quasars used are SDSS J1148+5251 $z=6.42$ (Willott et al. 2003), SDSS J1623+3112 $z=6.25$ (Jiang et al. 2007), SDSS J0005-0006 $z=5.85$, SDSS J0836+0054 $z=5.81$, SDSS $\mathrm{J} 1030+0524 z=6.31$, SDSS J1306+0356 $z=6.02$, SDSS J1411+1217 $z=5.90$ (Kurk et al. 2007) and 
SDSS J0303-0019 $z=6.08$ (Kurk et al. 2009). We take the values of $L_{3000}$ and $\mathrm{Mg}$ II FWHM from these papers and use the above equation to calculate the black hole mass in a consistent manner. Even though this is not a complete sample of SDSS $z \approx 6$ quasars, the target selection was mostly done on the basis of redshift and observability, so we do not expect any selection effects from the incompleteness. Thus our total sample consists of nine CFHQS quasars and eight SDSS quasars which span a factor of 20 in luminosity.

In Figure 5 we plot the Mg II FWHM and $M_{\mathrm{BH}}$ against $L_{3000}$ for these 17 quasars. It is immediately apparent that there is a strong positive correlation between FWHM and $L_{3000}$. This holds for both quasar surveys: the two high luminosity CFHQS quasars have relatively high FWHM and the two moderate luminosity SDSS quasars from the SDSS deep stripe (Jiang et al. 2008) have low FWHM (as previously discussed by Kurk et al. 2009). This strong correlation is particularly interesting because it is not seen at lower redshifts (Fine et al. 2008; Shen et al. 2008) where there is essentially no change in FWHM with luminosity. The fact that there was no correlation of FWHM with luminosity could cast doubt on the reliability of the virial black hole mass estimation method since correlations between $M_{\mathrm{BH}}$ and luminosity are driven by the fact that luminosity enters the equation used to estimate $M_{\mathrm{BH}}$. The reason that, at lower redshifts, there is no correlation between FWHM and luminosity is because the quasars are accreting at a very wide range of Eddington ratios (Shen et al. 2008). As we are about to show, this is not the case at $z=6$.

The Eddington luminosity is defined as the maximum luminosity attainable due to outward radiation pressure acting on infalling material and is $L_{\mathrm{Edd}}=1.3 \times$ $10^{31}\left(M_{\mathrm{BH}} / M_{\odot}\right) \mathrm{W}$. The observed monochromatic luminosity $L_{3000}$ is only a fraction of the total electromagnetic luminosity coming from the quasar, so a bolometric correction is applied to calculate the bolometric luminosity, $L_{\text {Bol }}$, from $L_{3000}$. We use a bolometric correction factor of 6.0 , consistent with previous work (Richards et al. 2006; Jiang et al. 2006). The ratio of the bolometric luminosity to the Eddington luminosity for a given black hole mass is often called the Eddington ratio and is defined as $\lambda \equiv L_{\mathrm{Bol}} / L_{\mathrm{Edd}}$.

The right panel of Figure 5 plots $M_{\mathrm{BH}}$ against $L_{3000}$. There is a strong positive correlation showing that more luminous quasars have greater black hole masses. The black hole masses in these $z=6$ quasars range from just under $10^{8} M_{\odot}$ up to almost $10^{10} M_{\odot}$. Also shown are lines for Eddington ratios of $\lambda=0.1$ and $\lambda=1$. The $z=6$ quasars are tightly clustered around the $\lambda=1$ line showing that they are almost all accreting at the Eddington limit. There are no quasars with $\lambda=0.1$ which are common at lower redshifts. The distribution of $\lambda$ will be discussed further in the following section. Note that some quasars appear to exceed the Eddington limit, although only by up to a factor of 3 .

Figure 5 highlights why the observation of low luminosity quasars at $z=6$ is so important. In the local universe (Shankar et al. 2009) and at the peak of quasar activity at $1<z<3$ (McLure \& Dunlop 2004; Shen et al. 2008; Vestergaard \& Osmer 2009) there appears to be a maximum black hole mass of $10^{10} M_{\odot}$, likely due to feedback effects on black hole growth in the most mas- sive galaxies. The high luminosities of most $z \approx 6$ SDSS quasars mean that they cannot be accreting at $\lambda=0.1$ because this would necessitate $M_{\mathrm{BH}}>10^{10} M_{\odot}$. It is the CFHQS quasars, with more moderate luminosities, that could be powered either by $\sim 10^{9} M_{\odot}$ black holes with $\lambda=0.1$ or $\sim 10^{8} M_{\odot}$ black holes with $\lambda=1$. Our results show that the latter is true.

\subsection{The Eddington ratio distribution}

The distribution of Eddington ratios, $\lambda$, in quasars is an important constraint on models of quasar activity and black hole growth. Studies up to $z=4$ show that the $\lambda$ distribution in luminosity and redshift bins is usually a lognormal which shifts to higher $\lambda$ and narrows at higher luminosities (Kollmeier et al. 2006; Shen et al. 2008). The narrowing of the distribution at high luminosity is likely related to the cutoff in the black hole mass function at $\sim 10^{10} M_{\odot}$. Shen et al. (2008) found that the most luminous quasars $\left(L_{\mathrm{Bol}}>10^{40} \mathrm{~W}\right)$ at $2<z<3$ have a typical $\lambda=0.25$ and dispersion of 0.23 dex. Some authors have discussed the Eddington ratio distribution as a function of black hole mass (Kollmeier et al. 2006; Netzer et al. 2007) since this relates more closely to model predictions. However, the selection effects imposed by flux-limited quasar samples coupled with the correlations of luminosity, $M_{\mathrm{BH}}$ and $\lambda$ have caused disagreement between the results of these studies.

The black hole masses of the $17 z=6$ quasars allow us to make the first determination of the $\lambda$ distribution at such a high redshift. We do not have sufficient quasars to determine the distribution as a function of luminosity, however inspection of Figure 5 suggests no luminositydependence. In Figure [6] we show the $\lambda$ distribution at $z=6$. The distribution can be approximated by a lognormal with peak $\lambda=1.07$ and dispersion 0.28 dex. Therefore the typical quasar at $z=6$ is observed to be accreting right at the Eddington limit and there is only a narrow $\lambda$ distribution.

The peak of the distribution is four times higher than for the most luminous quasars at $2<z<3$ found by Shen et al. (2008). However, the Shen et al. work used a smaller luminosity bin than in our study, so in order to make a detailed comparison with lower redshift we need to use a sample that is matched in luminosity. We use the McLure \& Dunlop (2004) Mg II FWHM line widths and luminosities for SDSS quasars at $1.7<z<2.1$. We use the same equation from Vestergaard \& Osmer (2009) as for the $z=6$ sample to determine $M_{\mathrm{BH}}$. In order to get luminosity-matched samples, we produce 10000 randomly drawn samples of 17 quasars from the $z=2$ SDSS sample matched to the $L_{3000}$ distribution of the $z=6$ sample. $\lambda$ is determined for each $z=2$ quasar using the same bolometric correction as the $z=6$ sample. The resulting $\lambda$ distribution at $z=2$ is shown in Figure6. The distribution peaks at significantly lower $\lambda$ than the $z=6$ sample $(\lambda=0.37)$ and is somewhat broader with dispersion 0.39 dex. A Kolmogorov-Smirnov test shows a probability of $10^{-6}$ that these two samples are drawn from the same parent distribution. Our results at $z=2$ are slightly different to the results of Shen et al. (2008), likely due to the fact we have a broader luminosity range.

The fact that virial black hole masses are calculated by $M_{\mathrm{BH}} \propto \mathrm{FWHM}^{2} L^{0.5}$ and that the FWHM distribution is not a strong function of luminosity or redshift at 


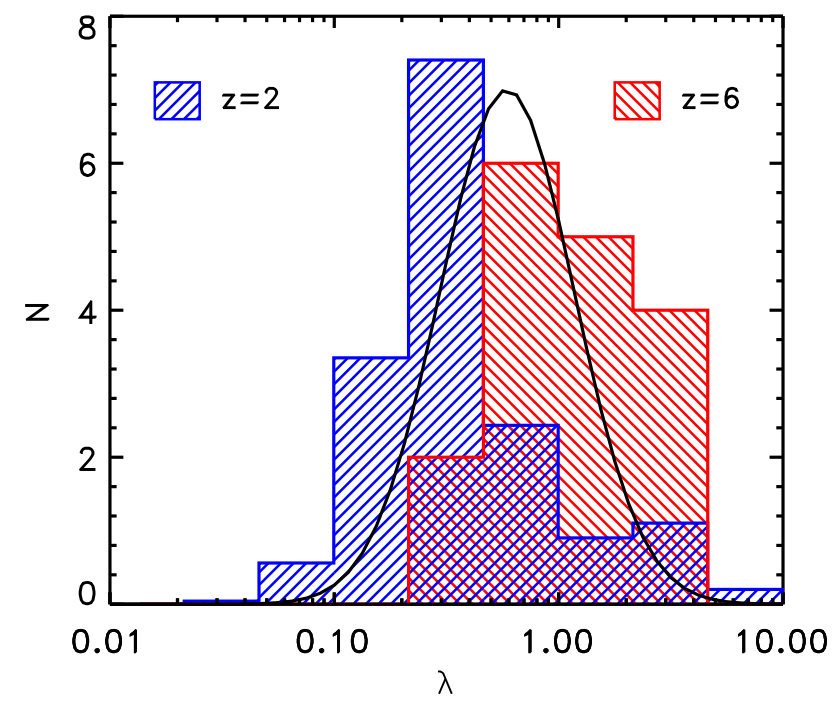

Figure 6. Observed distributions of the Eddington ratio for the luminosity-matched $z=2$ and $z=6$ quasar samples are shown as hatched histograms. The two distributions are clearly different showing that a much higher fraction of $z=6$ quasars are accreting at or just above the Eddington limit. The solid black line shows the intrinsic $\lambda$ distribution for $z=6$ black holes after accounting for the fact that high- $\lambda$ black holes are more likely to be found in flux-limited quasar samples than those with low values of $\lambda$.

$z<4$ has led skeptics to suggest that the virial black hole mass estimates are purely driven by the luminosity dependence. We have shown here a strong dependence of FWHM with luminosity at $z=6$ and a marked difference in the $\lambda$ distribution at $z=6$ compared to $z=2$. Given the theoretical expectation that one should see such a change at the highest redshifts (as we discuss further below) lends strong support to the use of virial black hole estimators at both high redshift and high luminosity (despite the fact the method is only calibrated via the luminosity - BLR radius relation at low redshift and low luminosity).

The observed distribution of $\lambda$ in Figure 6 is that of several magnitude-limited samples of quasars (SDSS and CFHQS). It is not therefore necessarily the same as the distribution of $\lambda$ for a volume-limited sample of $z=6$ black holes. Black holes with low accretion rates may not pass the quasar selection magnitude limits. One would expect the luminosity-selected sample to have a distribution of $\lambda$ shifted to higher values than the intrinsic $\lambda$ distribution of all black holes with a given $M_{\mathrm{BH}}{ }^{9}$.

To determine the expected magnitude of this effect, we have carried out simulations with a range of plausible black hole mass functions and Eddington ratio distributions. In each simulation we generate $10^{6}$ black holes in the mass range $3 \times 10^{7}-10^{10} M_{\odot}$ according to the mass function and assign a Eddington ratio randomly from a lognormal distribution. Excluding other causes of scatter (such as scatter in the bolometric correction) this allows us to determine the absolute magnitude of each black hole and then select absolute magnitude lim-

\footnotetext{
10 Note this is different to the bias noted by Shen et al. (2008) for the $\lambda$ distribution in a narrow luminosity bin which is biased too low due to scatter and the slope of the black hole mass function.
}

ited quasar samples. Due to the steepening of the black hole mass function at high masses (see Section [5), the absolute magnitude limit of the simulated sample is important. We chose the results for a $M_{1450}<-25$ sample because this is the typical magnitude of the CFHQS quasars. The observed distribution of $\lambda$ is still a lognormal and is shifted towards higher values from the input distribution by 0.26 dex. Therefore the intrinsic distribution which matches our observed $z=6$ distribution has a peak at $\lambda=0.60$. The intrinsic dispersion is only marginally broader than that observed (0.30 dex intrinsic compared to 0.28 dex observed). For brighter quasars, $M_{1450}<-26$, the results are very similar with a further positive shift in $\lambda$ of 0.05 dex, i.e about $10 \%$. From these simulations, we determine that the intrinsic distribution of $\lambda$ for a volume-limited sample of $z=6$ black holes would be a lognormal with peak $\lambda=0.6$ and dispersion 0.30 dex. This distribution, $P(\lambda)$, is plotted as a solid line on Figure 6] so the relatively small offset from the observed distribution can be appreciated. Although there may be some small dependence of the distribution with $M_{\mathrm{BH}}$, there is no evidence for a dependence in luminosity in Figure 5] so we assume no $M_{\mathrm{BH}}$ dependence. The observed $z=2$ Eddington ratio distribution in Figure 6 would be similarly offset from the intrinsic $z=2$ distribution due to the same selection effect being at work. We have not modeled this because we only need the $z=6$ intrinsic distribution for the analysis in this paper.

\subsection{Comparison with models, lifetimes, light curves and duty cycles}

In this section we compare the results above with several models for black hole accretion growth, concentrating on the distribution of Eddington ratios at $z=6$, implications for quasar lifetimes, light curves and the duty cycle. If the duty cycle is close to unity, i.e. there are no completely inactive black holes at $z=6$, then the solid curve in Figure 6] shows that essentially all $z=6$ black holes are accreting at $\lambda>0.1$ and half of them at $\lambda>0.6$.

Di Matteo et al. (2008) used hydrodynamic cosmological simulations to study the growth of black holes from $z=10$ to low redshift. The accretion rate onto the black hole was governed by Bondi-Hoyle accretion dependent upon the innermost gas density resolved by the simulations. Feedback from the quasar becomes important in their model when the black hole is accreting close to the Eddington limit and this leads to quasars only spending a short amount of their lifetimes at close to the Eddington limit (Springel et al. 2005). The $\lambda$ distribution at $z=6$ determined by Di Matteo et al. (2008) peaks at $\lambda=0.04$ and has a negligible fraction of the distribution at $\lambda>0.3$, which is clearly inconsistent with our distribution, unless our black hole masses are biased low by radiation pressure effects (Marconi et al. 2008), which we argue later is unlikely.

Sijacki et al. (2009) used a similar hydrodynamical simulation centred on a high density peak identified in a larger dark matter simulation. They chose a high density region in order to simulate the growth of a massive $\left(\sim 10^{9} M_{\odot}\right)$ black hole, such as those found by the SDSS. They adopted the same accretion and feedback prescriptions as in Di Matteo et al. (2008). At $z=6$ they found that all the massive $\left(>10^{7} M_{\odot}\right)$ black holes in this re- 
gion were highly accreting, with a typical $\lambda=0.5$. This is very similar to the results we have found. Our results are for both SDSS and CFHQS quasars and therefore likely probe a range of dark matter densities.

A similar re-simulation of a high-density region was carried out by Li et al. (2007). They found that during the initial growth phase at $z>7$ of the black holes which would eventually merge by $z=6$ to form a $>10^{9} M_{\odot}$ black hole, the black holes accreted at approximately the Eddington rate. As in the previous simulations, when the black hole has grown to a certain mass at the Eddington rate where feedback becomes important, the gas supply is shut off and the accretion rate drops dramatically.

All the above simulations contained almost no inactive black holes at $z=6$ (except for seed black holes which had experienced very little growth and are below the mass range that we are interested in of $\left.\sim 10^{7} M_{\odot}\right)$. Based on the plentiful gas supply in high-redshift galaxies, the high merger rate, and Bondi-Hoyle accretion, all black holes are expected to be active and the duty cycle is close to unity. This is fundamentally different to the situation at lower redshifts where many black holes have passed their peak accretion and their host galaxies either contain gas that will not cool or have been cleared of gas by feedback effects (e.g. Di Matteo et al. 2008).

The idea that quasar activity follows two phases; initial Eddington-limited accretion followed by decreased or intermittent sub-Eddington accretion has been around for a long time (Small \& Blandford 1992). Yu \& Lu (2008) used constraints from the local black hole mass function and the quasar luminosity function to show that the latter phase could be fit by quasar light curves where luminosity declines with time with a power-law index of -1.2 or -1.3 , as would be expected if the decline phase were due to the evolution of an isolated accretion disk (Canizzo et al. 1990). Hopkins \& Hernquist (2009) used the luminosity function and $\lambda$ distribution at $z<1$ to place constraints on quasar light curves and found a somewhat faster decline than the case of an isolated accretion disk, consistent with AGN feedback effects in hydrodynamical simulations of galaxy mergers (Hopkins et al. 2006a). They show that quasars at low redshift spend most of their time accreting at low Eddington rates $(\lambda \ll 0.1)$. Hopkins \& Hernquist also considered how well the derived quasar lifetimes could be translated into quasar light curves, but found that the data are degenerate between single long-lived accretion events taking several Gyr (of which only the first fraction of a Gyr has high $\lambda$ ) or light curves with multiple episodic short-lived accretion events. Observations such as the transverse proximity effect (Jakobsen et al. 2003; Worseck et al. 2007) and spectral aging studies of radio sources (Scheuer 1995) suggest high-accretion rate episodes of length $>10^{7}$ yrs and therefore provide only a weak limit on the number of potential episodes.

We have found that at $z \approx 6$ almost all moderate to high luminosity quasars are accreting at close to the Eddington rate. The absence of sub-Eddington quasars suggests that most quasars at this epoch are in the process of the exponential build-up of their central black holes and have not reached the later phase of quasar activity where the accretion rate declines. Only one or two of the quasars we have studied may be in the decline part of the light curve. Taking account of the bias in $\lambda$ due to the quasar sample magnitude limit, this leads us to conclude that at least $50 \%$ of black holes at $z=6$ are still in the Eddington-limited growth phase. In addition, the results of the simulations described above show that there are no inactive black holes at $z=6$, i.e. the duty cycle is close to unity. A similar conclusion was reached by Shankar et al. (2010) who showed that the strong clustering at $z>4$ measured by Shen et al. (2007) means quasars reside in high mass dark matter halos and (assuming a tight $M_{B H}-M_{\text {halo }}$ relation) must have a high duty cycle of $\approx 95 \%$ at $z=6$. These inferences on lifetimes and duty cycles are important for the next section where we determine the $z=6$ black hole mass function, because they mean that we are directly observing most of the high mass end of the black hole mass function at $z=6$ in quasars (with the likely exception of obscured quasars).

\section{BLACK HOLE MASS FUNCTION}

In the previous section we determined the intrinsic distribution of Eddington ratios for supermassive black holes at $z=6$ and also discussed their lifetimes and duty cycles. The fact that almost all black holes at $z=6$ appear to be active and accreting at rates approaching, or even exceeding, their Eddington rates means that it is a relatively trivial procedure to use the observed luminosity function of quasars to determine the underlying black hole mass distribution. This is in contrast to the situation at lower redshifts where a considerable fraction of black holes may have passed their peak activity or be going through a pause in activity and therefore one can either determine the active black hole mass function (Vestergaard et al. 2008; Shen et al. 2008) or one requires more model assumptions and constraints such as the Soltan argument to estimate the total black hole mass function (Somerville 2009; Shankar et al. 2009).

\subsection{Required inputs and assumptions}

The necessary assumptions for deriving the $z=6$ black hole mass function are:

- Observed $z=6$ quasar luminosity function

- Bolometric correction

- Correction of luminosity function for obscured quasars

- Correction for inactive black holes, i.e. duty cycle

- Eddington ratio distribution

We now discuss each of these in turn.

\subsubsection{Observed $z=6$ quasar luminosity function}

The luminosity function gives the space density of actively accreting black holes as a function of their luminosity. The best derivation of the luminosity function at $z=6$ (Willott et al. 2010) comes from optically-selected quasars from the SDSS and CFHQS surveys. Ideally, hard X-ray or mid-IR samples of quasars would be used since they account for some fraction of the opticallyobscured population. However, there are no AGN selected at X-ray or mid-IR wavelengths at this high a redshift. 


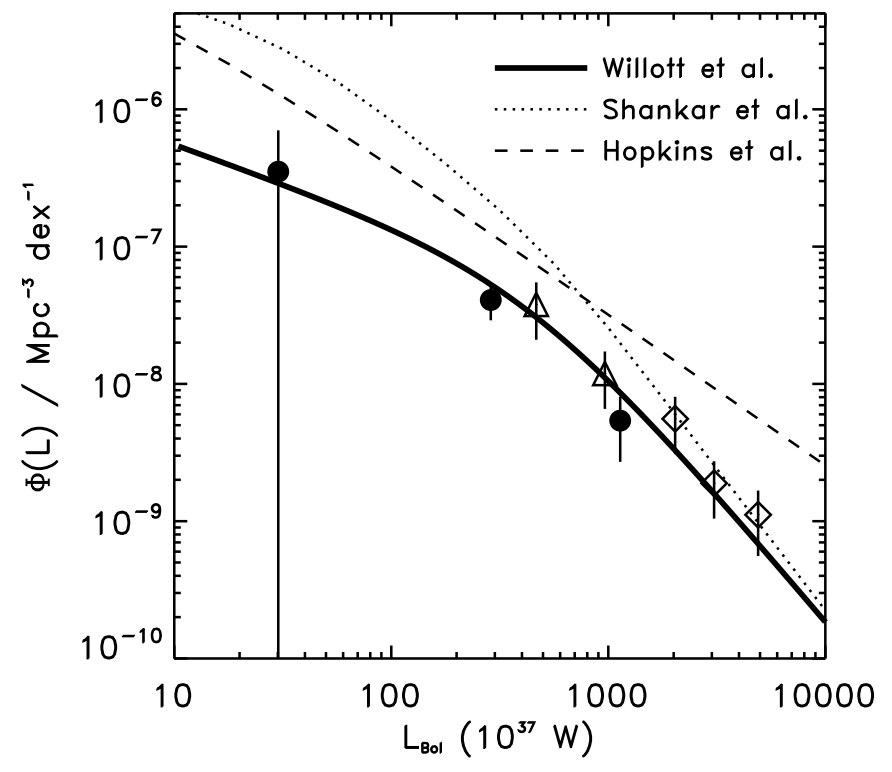

Figure 7. Comparison of bolometric quasar luminosity functions at $z=6$ corrected for the existence of obscured quasars as described in Section 5.1. The binned data points and thick black line are from the optical quasar luminosity function of Willott et al. (2010) using data from the SDSS main (diamonds - Fan et al. 2006), SDSS deep stripe (triangles - Jiang et al. 2009) and CFHQS (filled circles). Error bars on the binned data are only the Poisson errors on the quasar counts and do not include uncertainties in the bolometric correction or obscured AGN fraction. The dotted line is the luminosity function evolutionary model of Shankar et al. (2009) and the dashed line that of Hopkins et al. (2007). The CFHQS and SDSS deep stripe data are inconsistent with both model luminosity functions.

The luminosity function derived by Willott et al. (2010) used 40 quasars at $5.74<z<6.42$. These comprised 14 quasars from the SDSS main sample (Fan et al. 2006), 10 quasars from the SDSS deep stripe (Jiang et al. 2009) and 16 quasars from the CFHQS (Willott et al. 2010) giving a broad range in luminosity. The luminosity function was fit by a double power law model with best fit parameters of $\Phi\left(M_{1450}^{*}\right)=1.14 \times 10^{-8} \mathrm{Mpc}^{-3} \mathrm{mag}^{-1}$, break magnitude $M_{1450}^{*}=-25.13$ and bright end slope $\beta=-2.81$. A faint end slope of $\alpha=-1.5$ was assumed. Although there is considerable uncertainty on the values of these parameters, due to covariance of the parameters, the space density of optical quasars at $z=6$ is strongly constrained ( $1 \sigma$ uncertainty $<0.1 \mathrm{dex}$ ) over the range $-27.5<M_{1450}<-24.7$. The faintest quasar used, CFHQS J0216-0455 at $z=6.01$, has absolute magnitude $M_{1450}=-22.2$, but this being the only quasar at $M_{1450}>-24$ means the luminosity function is quite uncertain at low luminosities.

\subsubsection{Bolometric correction}

As in Section 4.1 we use a bolometric correction from the work of Richards et al. (2006) to convert between monochromatic UV luminosity and bolometric luminosity. Because the quasar luminosity function is defined at $1450 \AA$ we use a luminosity correction factor of 4.4 . The bolometric correction is not an important factor here because the black hole mass is directly determined from the virial mass estimator which uses the observed UV continuum luminosity. We therefore do not include any extra scatter due to the bolometric correction.

\subsubsection{Correction of luminosity function for obscured quasars}

The optical quasar luminosity function does not account for the population of actively accreting black holes which have their optical (in this case rest-frame far-UV) radiation absorbed by dust. In order to determine the full population of accreting black holes, one needs to correct for the missing population. The obscured AGN may be obscured by a geometrical torus as required by the simple unified scheme (Antonucci 1993; Urry \& Padovani 1995) or by dust in the quasar host galaxy (MartinezSansigre et al. 2005). It is now well established that low luminosity AGN are more frequently obscured than those of higher luminosity (Lawrence 1991; Ueda et al. 2003) and therefore we need to adopt a correction that is luminosity-dependent. Unfortunately, there is no evidence yet on the redshift dependence of the obscured fraction up to $z=6$. There appears to be no evolution in this fraction from $z=0$ to $z=2$ (Ueda et al. 2003). Therefore we take the low redshift absorbed AGN fraction as a function of luminosity as determined by Ueda et al. (2003). We also include Compton thick AGN following Shankar et al. (2009), although this only makes a modest further increase. Including obscured quasars raises the space density by a factor of 2 at $M_{1450}=-27.2$ and a factor of 3 at $M_{1450}=-20.7$.

Figure 7 shows the bolometric luminosity function of Willott et al. (2010) using the bolometric correction and correction for obscured quasars described above. Also plotted are $z=6$ bolometric luminosity function models from Hopkins et al. (2007) and Shankar et al. (2009; based on the compilation of Shankar \& Mathur 2007). Both the Shankar et al. and Hopkins et al. luminosity functions over-predicts the numbers of quasars compared to the observations. This is discussed further in Section 5.1 .6 .

\subsubsection{Correction for inactive black holes, i.e. duty cycle}

As discussed at the end of Section 4.3, there are both theoretical and observational arguments that, at $z=6$, the duty cycle of black holes is very high. We adopt a default duty cycle of 0.75 and consider a plausible range of 0.5 to 1 .

\subsubsection{Eddington ratio distribution}

Using a constant bolometric correction means that a given black hole mass maps directly on to a value of $M_{1450}$ for black holes accreting at exactly the Eddington limit. The Eddington ratio distribution, $P(\lambda)$, is used to map a given black hole mass onto a range of $M_{1450}$. We use the intrinsic Eddington ratio distribution at $z=6$ determined in Section 4 and plotted in Figure 6] as a solid line which is a lognormal centred at $\lambda=0.6$ with standard deviation of 0.30 dex. We do not apply a cutoff above the Eddington limit, as quasars are observed to have values up to $\lambda=10$ (although of course some of this is due to scatter in the measurements and correlations used to determine black hole masses). In any case, this distribution only puts $4 \%$ of black holes at $\lambda>2$. We do not have sufficient data to determine if there is any black hole mass dependence of $P(\lambda)$. At 
the lowest luminosities and black hole masses, we do not have any quasars with black hole masses from the $\mathrm{Mg}$ II line so we assume that $P(\lambda)$ is the same at all luminosities. We note that the lowest luminosity quasar in the CFHQS sample, CFHQS J0216-0455, does have a very narrow Ly $\alpha$ line which would have an intrinsic FWHM of $1600 \mathrm{~km} \mathrm{~s}^{-1}$ correcting for blue wing IGM absorption (Willott et al. 2009). If Mg II has a similar width, this quasar would be accreting at the Eddington limit. We assumed the population of obscured AGN has the same Eddington ratio distribution as that of the unobscured population.

\subsubsection{Deriving the black hole mass function}

Rather than attempt to invert the observed luminosity function of Willott et al. (2010) to determine the black hole mass function, we instead use model black hole mass functions to produce luminosity functions which are then fit to the same quasar samples as Willott et al. (2010). Therefore much of the fitting procedure is the same as the luminosity function fitting and we refer the interested reader to that paper for full details. The best-fit is determined via the maximum likelihood method using amoeba parameter optimization.

The main effect of including scatter in the conversion of black hole mass to quasar luminosity is to flatten the bright end slope of the luminosity function compared to the slope for the black hole mass function. Fits to the black hole mass function were attempted using either double power laws or Schechter functions. The double power law fits produced a very steep high mass end and similar likelihood to the Schechter function fits. Therefore we adopted the Schechter function for the black hole mass function since it required one fewer parameter to be fit. The Schechter function form is favoured theoretically because there should be a sharp cutoff in mass of the most massive black holes, as is observed in the local black hole mass distribution (e.g. Shankar et al. 2009) and the $z=2$ black hole mass distribution (Vestergaard et al. 2008), which is plausibly due to feedback in the most massive galaxies, and the limited cosmic time available for black hole growth by $z=6$ (Volonteri \& Rees 2005).

The process then is to take the model black hole mass function, convolve with $P(\lambda)$, convert to luminosity and absolute magnitude using the bolometric correction, correct for obscured AGN and the duty cycle to generate a model luminosity function. This model luminosity function is compared to the data and the parameters of the black hole mass function optimized to generate the best fit that maximizes the likelihood. The $z=6$ black hole mass function, $\Phi\left(M_{\mathrm{BH}}\right)$, assuming a duty cycle of 0.75 , has best fit parameters of $\Phi\left(M_{\mathrm{BH}}\right)^{*}=1.23 \times 10^{-8} \mathrm{Mpc}^{-3} \mathrm{dex}^{-1}$, characteristic mass $M_{\mathrm{BH}}^{*}=2.24 \times 10^{9} M_{\odot}$ and faint end slope $\alpha=-1.03$.

To determine the plausible range of black hole mass functions consistent with the data, we employ bootstrap resampling as described in Willott et al. (2010). We generate 100 samples of the data and determine the best fit to each. To account for the uncertainty in the duty cycle at $z=6$ we take the duty cycle as a uniformly distributed random number between 0.5 and 1 for each resample. In this way, we generate 100 plausible black

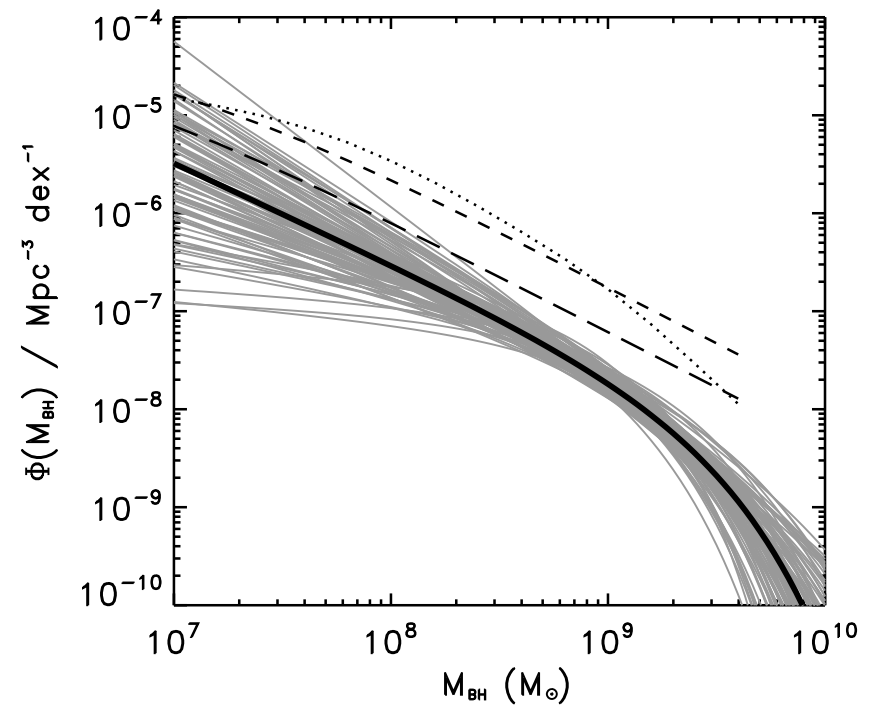

Figure 8. $z=6$ black hole mass function determined in Section [5] The thick solid black line is the best-fit to the data. The thin gray lines are the 100 bootstrap resamples consistent with the data. The black hole mass function is most strongly constrained at $M_{\mathrm{BH}}>10^{8} M_{\odot}$ and at $M_{\mathrm{BH}}<3 \times 10^{9} M_{\odot}$. Also plotted are $z=6$ black hole mass functions determined from the evolutionary models of Shankar et al. (2009) assuming their luminosity function and reference model $(\epsilon=0.065, \lambda=0.4, z=6$ duty cycle $=0.5)$ (dotted line) and assuming the Hopkins et al. (2007) luminosity function with the reference model (short-dashed line) and also with different parameters of $\epsilon=0.09, \lambda=1, z=6$ duty cycle $=0.5$ (long-dashed line).

hole mass functions.

Figure 8 shows the 100 bootstrap resampled mass functions as overlapping gray lines and the best fit as a thick black line. The mass function is most strongly constrained close to $10^{9} M_{\odot}$. This is not surprising given the range of black hole masses of SDSS and CFHQS quasars shown in Figure 5. There is considerable divergence at $M_{\mathrm{BH}}<10^{8} M_{\odot}$ and at $M_{\mathrm{BH}}>3 \times 10^{9} M_{\odot}$ due to the few very low or very high luminosity quasars used to derive the luminosity function.

Also shown on Figure 8 are three determinations of the $z=6$ black hole mass function by Shankar et al. (2009). These were generated by self-consistent models which fit the evolving AGN luminosity function, local black hole mass function and X-ray background and are taken from their Table 3. One curve is for the Shankar et al. reference model and the other two are using the same model but fitting the Hopkins et al. (2007) luminosity function rather than that compiled by Shankar et al. The black hole mass function determined by us is considerably lower than all three of the Shankar et al. models, by factors ranging from 3 to 10 . We will next investigate the reasons for these differences.

Most cosmic accretion occurs at $z \ll 6$ and therefore the local black hole mass function and cosmic X-ray background are not strong constraints on the $z=6$ black hole mass functions derived by Shankar et al. (2009). The main reason for the difference between our result and theirs comes from their luminosity functions (Figure 7) and parameters used to convert between luminosity and black hole mass. The luminosity function derived by Shankar et al. (2009) agrees well with the bright end 
of the $z=6$ luminosity function of Fan et al. (2004) but is significantly steeper than that determined by Willott et al. (2010). Given that Willott et al. (2010) also found a slightly lower bright end normalization, means that our best-fit luminosity function is 3 times lower than the reference model of Shankar et al. (averaged over $-28<M<-23)$. The other luminosity function used by Shankar et al. is that of Hopkins et al. (2007). As seen in Figure 7 this function gives fewer moderate luminosity quasars than the Shankar et al. luminosity function, but more high luminosity ones. This can be seen also for the black hole mass functions in Figure 8. The Hopkins et al. luminosity function is on average 4 times higher than that of Willott et al. averaged over $-28<M<-23$. At moderate quasar luminosities $(-26<M<-24)$ the space densities of both the Shankar et al. and Hopkins et al. luminosity functions are strongly ruled out by the data of Willott et al. Therefore differing luminosity functions account for a large part of the difference between our black hole mass function and that of Shankar et al.

The rest of the difference can be accounted for by the parameters used to translate black hole masses to luminosities and the duty cycle. The relevant parameters for the reference model of Shankar et al. (2009) are accretion efficiency $\epsilon=0.065$, constant Eddington ratio $\lambda=0.4$ and duty cycle at $z=6$ of 0.5 . Only the duty cycle evolves with redshift in the reference model. In comparison, our analysis assumes $\epsilon=0.09$, a distribution of $\lambda$ peaked at 0.6 and a duty cycle drawn from a uniform distribution between 0.5 and 1 for the bootstrap resamples. The long-dashed line shows the model using the Hopkins et al. (2007) luminosity function with different parameters $(\epsilon=0.09, \lambda=1)$, which were required to fit the local black hole mass function. One can see that the effect of using these parameters, which are similar to ours, shifts the black hole mass function lower by a factor of $\sim 3$. Therefore we see that the differences in duty cycle, accretion efficiency, Eddington ratio and luminosity functions cause the differences between our results and those of Shankar et al. (2009). Both the Eddington ratio and luminosity function have been determined by us and therefore are an improvement on previous work. The duty cycle and accretion efficiency are still very much unknown.

The hydrodynamic simulations of Di Matteo et al. (2008) make predictions for the evolution of the black hole mass function. Due to the relatively small simulation box size, their results are only valid for black holes up to $M_{\mathrm{BH}} \sim 10^{8} M_{\odot}$. They predict a space density of $z=6$ black holes a factor of $\sim 100$ greater than we do. In large part this is due to their very much lower assumed accretion rates discussed in Section 4.3. Our quasars are fewer in number but growing much more rapidly than those in their simulations.

Marconi et al. (2008) derived corrections to virial black hole mass estimates based on the effect that radiation pressure has on the gas velocities. They showed that close to the Eddington limit this could have a very large effect with black hole masses being underestimated by a factor of $\sim 10$ assuming the BLR cloud column density is $N_{\mathrm{H}}=10^{23} \mathrm{~cm}^{-2}$. We did not use the Marconi et al. corrections due to the unknown value of $N_{\mathrm{H}}$. We note that because the $z=6$ quasars appear to be accreting close to the Eddington limit, this correction would be so large that it would shift the entire black hole mass function to higher masses by 1 dex. This would then give the exponential cutoff in the mass function at $M_{\mathrm{BH}} \sim$ $3 \times 10^{10} M_{\odot}$. It does not seem plausible that the mass function at $z=6$ would contain such a large number of $>10^{10} M_{\odot}$ black holes given the lack of such black holes at low redshift (Tundo et al. 2007), at $z=2$ (McLure \& Dunlop 2004; Vestergaard \& Osmer 2009), self-regulation arguments (Natarajan \& Treister 2009) and the difficulty of forming them in the cosmological time available by $z=6$ (Volonteri \& Rees 2006). The issue of whether or not one should make radiation pressure modifications to virial black hole mass estimates is also discussed by Peterson (2010).

\subsection{Global evolution of black hole and stellar mass functions}

The $z=6$ black hole mass function we have derived can be compared to the present day black hole mass function in order to determine the total black hole growth between $~ 1$ Gyr after the Big Bang to today. Locally, most black holes are inactive and measurement of their masses via dynamical tracers such as gas and stars have only been performed for about 50 of the closest galaxies (Gultekin et al. 2009). Given the small scatter in scaling relations such as the $M_{\mathrm{BH}}-\sigma$ and $M_{\mathrm{BH}}-L$ relations, it is common to derive the local black hole mass function by using the observed velocity dispersion function or luminosity function of galaxies in conjunction with scaling relations (see Tundo et al. 2007 for a comparison of these methods). We use the range of plausible local black hole mass functions determined from analysis by Shankar et al. (2009) of several such studies (plotted as a gray band in Figure 9).

The local and $z=6$ black hole mass functions shown in Figure 9 have rather similar shapes. The local function steepens above a somewhat lower mass of $\sim 10^{9} M_{\odot}$ than the $z=6$ function. There is also some evidence for a steeper $z=6$ function at $10^{7} M_{\odot}<M_{\mathrm{BH}}<3 \times 10^{8} M_{\odot}$, although the $z=6$ function is not well constrained at the low mass end, due to few low-luminosity quasars and lack of $\lambda$ measurements for them. The overall normalization of the $z=6$ black hole mass function is $\sim 10^{-4}$ times that at $z=0$, highlighting just how rare black holes are at this early epoch.

Due to the existence of local scaling relations between black holes and galaxy properties and the implications for the importance of black holes in galaxy evolution, it is interesting to compare this evolution of the black hole mass function with the evolution of the mass which has formed into stars within galaxies. For the local stellar mass function we use the $z<0.05$ determination using the SDSS New York University Value-Added Galaxy Catalog (Baldry et al. 2008). For the stellar mass function at $z=6$ we use that determined via star formation history SED fits to $z \approx 6$ Lyman break galaxies with deep Spitzer photometry by Stark et al. (2009). These data start to become incomplete at $M_{\text {stellar }}<10^{10} M_{\odot}$ and have a large uncertainty at $M_{\text {stellar }}=10^{11} M_{\odot}$ due to the small volumes probed by these Lyman break surveys.

In Figure 9 we plot the $z=0$ and $z=6$ stellar mass functions along with the black hole mass functions. The upper axis shows the stellar mass and it has been shifted horizontally by $2 \times 10^{-3}$ compared to the black hole mass 


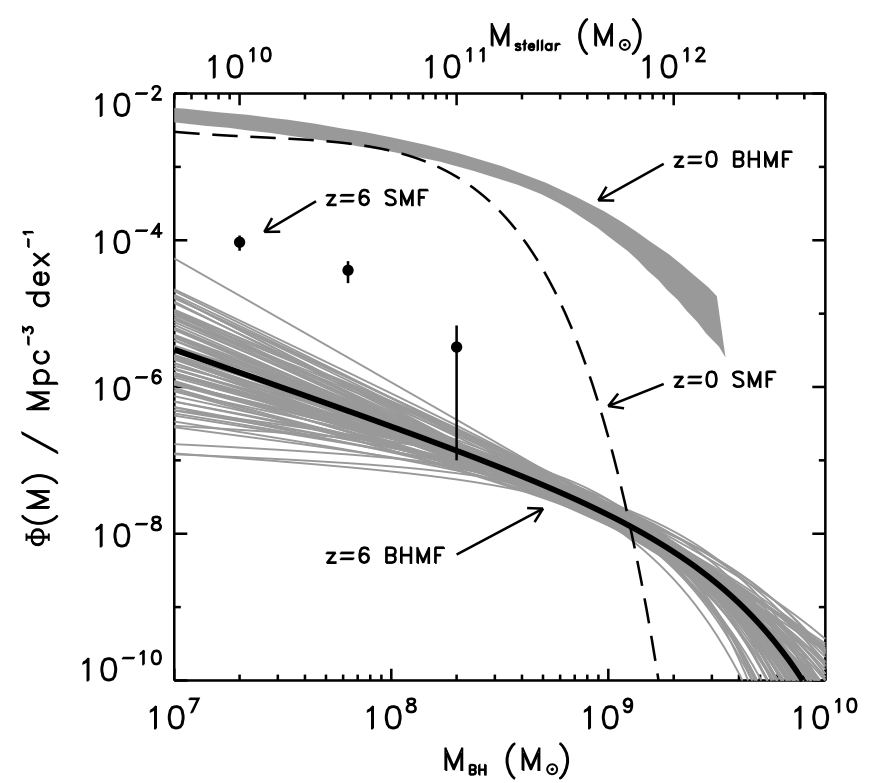

Figure 9. The black hole mass function at $z=6(z=6 \mathrm{BHMF}$; thick black line and grey curves as in Figure 8 compared with the local black hole mass function ( $z=0$ BHMF; grey band - Shankar et al. 2009), the local stellar mass function $(z=0 \mathrm{SMF}$; dashed line - Baldry et al. 2008) and the $z=6$ stellar mass function $(z=6 \mathrm{SMF}$; circles with error bars - Stark et al. 2009). The two stellar mass functions have been shifted horizontally by a factor of $2 \times 10^{-3}$, as indicated in the upper axis, to match up the space densities of galaxies and black holes at $z=0$.

axis. This factor is similar to the factors of $1.4 \times 10^{-3}$ (Haring \& Rix 2004) and $2 \times 10^{-3}$ (Tundo et al. 2007) determined from fits to the local $M_{\mathrm{BH}}-M_{\text {bulge }}$ relation and approximately matches the space densities of galaxies and black holes at $z=0$ in Figure 9 .

Figure 9 shows the rather remarkable result that whereas the stellar mass density evolution from $z=6$ to $z=0$ is $\sim 100$, the black hole mass density evolution over this same time period is $\sim 10^{4}$, i.e. 100 times greater. This is observed to hold over about one order of magnitude in mass. At low black hole masses and high stellar masses the comparison is difficult because of the vastly different volume surveys undertaken for quasars and galaxies at $z=6$. At $M_{\text {stellar }}=10^{11} M_{\odot}$, the very large uncertainty in stellar mass density makes it plausible that it matches that of black holes. Larger area optical to mid-IR surveys for $\sim 10^{11} M_{\odot}$ galaxies at $z \approx 6$ are needed to address whether there is an exponential decline in the stellar mass function at this mass which would match the space density of $M_{\mathrm{BH}}=2 \times 10^{8} M_{\odot}$ black holes.

We identify two possible reasons for this big difference between the mass accreted on to black holes and that which has formed stars by $z=6$. Eddington-limited accretion occurs exponentially so at early times when the black hole masses are small, there is a limit to how fast they can grow. In comparison, star formation has no similar restriction and in fact the high densities in the early universe may actually have enhanced the star formation rate (e.g. Granato et al. 2004). Another possibility is that only a fraction of galaxies were seeded with massive $\gtrsim 10^{4} M_{\odot}$ black holes. Most theoretical attempts to account for the most massive black holes observed at $z=6$ have shown that seed black holes of this mass are required if accretion obeys the Eddington limit (Yoo \& Miralda-Escudé 2004; Li et al. 2007; Sijacki et al. 2009). Therefore the finding of a large discrepancy between the space densities of black holes and galaxies may be indicating that only a fraction of galaxies start with massive seeds. The less massive seeds in other galaxies will catch up by the peak in quasar activity at $z \sim 2$.

This result is in contrast to observations of the evolution of the $M_{\mathrm{BH}}-M_{\text {bulge }}$ relation. In several studies it has been found that for AGN-selected samples, the ratio of $M_{\mathrm{BH}}$ to $M_{\text {bulge }}$ evolves to higher values at higher redshift (Walter et al. 2004; Peng et al. 2006; McLure et al. 2006; Riechers et al. 2008; Merloni et al. 2010; Wang et al. 2010). It is important to note that due to scatter in the $M_{\mathrm{BH}}$ to $M_{\text {bulge }}$ (or $M_{\text {halo }}$ ) relation combined with steep mass functions, luminous quasars will preferentially be found in lower mass hosts than would be expected based on the relation for a volume-limited sample of galaxies (Willott et al. 2005; Lauer et al. 2007). Somerville (2009) and Merloni et al. (2010) show that this bias could almost completely account for the observed evolution of the relation and that up to $z=2$ there is at most a factor of two of positive evolution in $M_{\mathrm{BH}} / M_{\text {bulge }}$. Other studies, selecting galaxies to be gas-rich starbursts without luminous AGN (Borys et al. 2005; Alexander et al. 2008), actually show negative evolution in $M_{\mathrm{BH}} / M_{\text {bulge }}$ highlighting how important selection effects are for distinct galaxy populations. Note that our results are not necessarily in conflict with the molecular gas observations of $z \sim 6$ quasars that show they have high ratios of $M_{\mathrm{BH}}$ to $M_{\text {bulge }}$ (Walter et al. 2004; Riechers et al. 2008; Wang et al. 2010). The point is that there are many more galaxies with very low ratios of $M_{\mathrm{BH}}$ to $M_{\text {bulge }}$ which are not observed in quasar samples.

Attempts to study the theoretical global evolution in star formation versus black hole growth have been carried out by Robertson et al. (2006), Hopkins et al. (2006b) and Shankar et al. (2009). These show little evolution in the ratio, however they mostly do not go up to $z=6$ or are poorly constrained there. Di Matteo et al. (2008) consider the evolution of the stellar mass density and black hole mass density in their simulations. They find that although there is little evolution from the local ratio up to $z=2$, there is considerable evolution from $z=2$ to $z=6$ of nearly a factor of 10 (in the sense that the black hole mass density declines more rapidly). Their results are similar to ours, but with less rapid evolution, which can be explained because their simulations have a lower typical $\lambda$ than we observe and therefore a higher black hole mass function. Li et al. (2007) found in their simulations that the total stellar mass formed in the galaxies which would merge to become a $z=6$ SDSS host galaxy led the total black hole mass by factors of 10 to 100 at early times $(8<z<14)$. Although not discussed in their paper, the likely reason for this behaviour is the Eddington limit which restricts the very early growth of the black hole mass. Lamastra et al. (2010) use a semianalytic cosmological model to predict positive evolution of $M_{\mathrm{BH}} / M_{\text {bulge }}$ by a factor of 3 for the global population up to $z=7$ which they explain as due to very efficient black hole growth at early times. Their work suggests a ratio of black hole to stellar mass density $\sim 300$ times 
greater than we have found!

Our results hinge upon the assertions that the quasar duty cycle is very high at $z=6$, there is little evolution in the fraction of obscured AGN and therefore we have identified all the black holes as AGN. The $z=6$ Lyman break galaxies with stellar masses $>10^{10} M_{\odot}$ are about 100 times more common than low-luminosity quasars. It is possible that these galaxies contain inactive or obscured black holes and we have underestimated the true space density of black holes. We did account for a factor of $2-3$ of obscured quasars, as observed at lower redshift, but perhaps this factor evolves dramatically at the highest redshifts due to the dusty nature of these young, forming host galaxies. Mid-IR-selected AGN samples do show an increase in the obscured fraction at $z>3$ (M. Lacy, priv. comm.). Although there are observations requiring large dust masses in many $z \sim 6$ quasar host galaxies (Wang et al. 2008), the Lyman break-selected $z \sim 6$ galaxies have very low dust extinction based on their UV spectral slopes (Bouwens et al. 2009).

At $z \sim 2-3$, about $3-5 \%$ of Lyman break galaxies contain evidence for weak AGN (Steidel et al. 2002; Reddy et al. 2006). Spectra of $z=6$ galaxies show a high incidence of narrow Ly $\alpha$ emission lines (Stanway et al. 2007; Ouchi et a. 2008), but most higher ionization metal lines which could be used to test for AGN lie in the near-IR. The NIRSpec instrument on the James Webb Space Telescope will be able to detect weak high ionization lines and/or weak broad Balmer lines in these galaxies to determine if they do host hidden AGN. Another potential method of searching for obscured AGN activity is mid-IR imaging of the hot dust with the MIRI instrument.

\section{CONCLUSIONS}

We have presented new near-IR spectroscopy for nine of the most distant known, moderate luminosity quasars. These data have been used to estimate virial black hole masses based on the Mg II linewidth and UV luminosity. Adding in published data on more luminous quasars we obtain a sample of 17 quasars at $z \gtrsim 6$. Our results and conclusions can be summarized as follows.

- We observe a positive correlation between the Mg II line FWHM and UV continuum luminosity. Such a correlation is usually absent from lower redshift samples due to the broad range of Eddington ratios at lower redshift (Fine et al. 2008; Shen et al. 2008). The existence of this correlation at high-redshift provides support for the validity of the virial linewidth estimator.

- There is a linear correlation between black hole mass and UV continuum luminosity and the quasars are accreting close to the Eddington limit. The distribution of observed Eddington ratios is a lognormal centred on $\lambda=1.07$ with dispersion 0.28 dex. This distribution is significantly different from that of a luminosity-matched $z=2$ sample which has a distribution centred on $\lambda=0.37$ with a broader dispersion of 0.39 dex. Accounting for selection effects due to the quasar sample magnitude limits, we determine the intrinsic Eddington ratio distribution for a volume-limited sample of black holes at $z=6$. This distribution is a lognormal centred on $\lambda=0.60$ with dispersion 0.30 dex. The Eddington ratio distribution we find at $z=6$ is consistent with the results of simulations of highdensity peaks in the early dark matter distribution (Li et al. 2007; Sijacki et a. 2009).

- The implication of these results is that at $z=6$ all the quasars we are observing are still in their initial exponential growth phases due to their young host galaxies and a plentiful gas supply. Combining the Eddington ratio distribution with the assumption of a high duty cycle and the observed quasar luminosity function of Willott et al. (2010) we can derive the black hole mass function. Note that this is a much harder problem at low redshifts where the Eddington ratio distribution is broader and the duty cycle is unknown. The resulting black hole mass function is factors of 3 to 10 below previous estimates (Shankar et al. 2009) due to a lower luminosity function normalization, accretion radiative efficiency, duty cycle and higher Eddington ratios.

- The evolution in the black hole mass function from $z=6$ to $z=0$ is a factor of $\sim 10^{4}$. This is much greater than the $\sim 10^{2}$ increase in the stellar mass function over the same redshift interval. This means that the stars in galaxies were formed much more rapidly at high redshift than black holes grew, presumably due either to the limited rate at which black holes can grow due to radiation pressure or that only a small fraction of galaxies had massive initial black hole seeds.

Thanks to Ross McLure, Francesco Shankar and Dan Stark for providing data in electronic form and the anonymous referee for useful suggestions for improvements. Based on observations obtained with MegaPrime/MegaCam, a joint project of CFHT and CEA/DAPNIA, at the Canada-France-Hawaii Telescope (CFHT) which is operated by the National Research Council (NRC) of Canada, the Institut National des Sciences de l'Univers of the Centre National de la Recherche Scientifique (CNRS) of France, and the University of Hawaii. This work is based in part on data products produced at TERAPIX and the Canadian Astronomy Data Centre as part of the Canada-France-Hawaii Telescope Legacy Survey, a collaborative project of NRC and CNRS. Based on observations obtained at the Gemini Observatory, which is operated by the Association of Universities for Research in Astronomy, Inc., under a cooperative agreement with the NSF on behalf of the Gemini partnership: the National Science Foundation (United States), the Particle Physics and Astronomy Research Council (United Kingdom), the National Research Council (Canada), CONICYT (Chile), the Australian Research Council (Australia), CNPq (Brazil) and CONICET (Argentina). This paper uses data from Gemini programs GS-2006A-Q-16, GS-2009B-Q-25, GN-2007AQ-201, GN-2007B-Q-35, GN-2008B-Q-43, GN-2009B-Q33 and GN-2009B-DD-5. Based on observations made with the ESO New Technology Telescope at the La Silla Observatory. 


\section{REFERENCES}

Alexander, D. M., et al. 2008, AJ, 135, 1968

Antonucci, R. R. J. 1993, ARAA, 31, 473

Baldry, I. K., Glazebrook, K., \& Driver, S. P., 2008, MNRAS, 388,945

Bentz, M. C., Peterson, B. M., Netzer, H., Pogge, R. W., \& Vestergaard, M. 2009, ApJ, 697, 160

Bolton, J. S., \& Haehnelt, M. G. 2007, MNRAS, 382, 325

Borys, C., Smail, I., Chapman, S. C., Blain, A. W., Alexander, D. M., \& Ivison, R. J. 2005, ApJ, 635, 853

Bouwens, R. J., et al. 2009, ApJ, 705, 936

Cannizzo, J. K., Lee, H. M., \& Goodman, J. 1990, ApJ, 351, 38

Carilli, C. L., et al. 2010, ApJL, in press, arXiv:1003.0016

Cen, R., \& Haiman, Z. 2000, ApJ, 542, L75

Croton, D. J., et al. 2006, MNRAS, 365, 11

Denney, K., Peterson, B. M., Dietrich, M., Vestergaard, M., \& Bentz, M. C. 2009, ApJ, 692, 246

Diamond-Stanic, A. et al. 2009, ApJ, 699, 782

Di Matteo T., Springel V., \& Hernquist L. 2005, Nature, 433, 604

Di Matteo T., Colberg J., Springel V., Hernquist L., \& Sijacki D. 2008, ApJ, 676, 33

Fan, X., et al. 2003, AJ, 125, 1649

Fan, X., et al. 2004, AJ, 128, 515

Fan, X., et al. 2006, AJ, 132, 117

Ferrarese, L., \& Merritt D. 2000, ApJ, 539, L9

Fine, S. et al. 2008, MNRAS, 390, 1413

Gebhardt, K. et al. 2000, Ap.J, 539, L13

Granato, G. L., De Zotti, G., Silva, L., Bressan, A., \& Danese, L. 2004, ApJ, 600, 580

Gultekin, K. et al. 2009, ApJ, 698, 1986

Haring, N. \& Rix, H.-W. 2004, ApJ, 604, L89

Hopkins, P. F., Hernquist, L., Cox, T. J., Di Matteo, T., Robertson, B., \& Springel, V. 2006a, ApJS, 163, 1

Hopkins, P. F., Robertson, B., Krause, E., Hernquist, L., \& Cox, T. J. 2006b, ApJ, 652, 107

Hopkins, P. F., Richards, G. T., \& Hernquist, L. 2007, ApJ, 654, 731

Hopkins, P. F. \& Hernquist, L. 2009, ApJ, 698, 1550

Jakobsen, P., Jansen, R. A., Wagner, S., \& Reimers, D. 2003, A\&A, 397, 891

Jiang, L., et al. 2006, AJ, 132, 2127

Jiang, L., et al. 2007, AJ, 134, 1150

Jiang, L., et al. 2008, AJ, 135, 1057

Jiang, L., et al. 2009, AJ, 138, 305

Kaspi, S., Smith, P. S., Netzer, H., Maoz, D., Jannuzi, B. T., \& Giveon, U., 2000, ApJ, 533, 631

Kawakatu, N., \& Wada, K. 2009, ApJ, 706, 676

Kollmeier, J. A. et al. 2006, ApJ, 648, 128

Komatsu, E., et al., 2009, ApJS, 180, 330

Kurk, J. D., et al. 2007, ApJ, 669, 32

Kurk, J. D., Walter, F., Fan, X., Jiang, L., Jester, S., Rix, H.-W., Riechers, D. A. 2009, ApJ, 702, 833

Lamastra, A., Menci, N., Maiolino, R., Fiore, F., \& Merloni, A. 2010, MNRAS, 405, 29

Lauer, T. R., Tremaine, S., Richstone, D., \& Faber, S. M. 2007, ApJ, 670, 249

Lawrence, Ȧ. 1991, MNRAS, 252, 586

Li, Y., et al. 2007, ApJ, 665, 187

Magorrian, J., et al. 1998, AJ, 115, 2285

Marconi, A., Risaliti, G., Gilli, R., Hunt, L. K., Maiolino, R., \& Salvati, M. 2004, MNRAS, 351, 169

Marconi, A., Axon, D., Maiolino, R., Nagao, T., Pastorini, G.

Pietrini, P., Robinson, A., \& Torricelli, G. 2008, ApJ, 678, 693

Martinez-Sansigre, A., et al. 2005, Nature, 436, 666

Mathur, S., \& Grupe, D. 2005, ApJ, 633, 688

McLure, R. J. \& Dunlop, J. S. 2004, MNRAS, 352, 1390

McLure, R. J., Jarvis, M. J., Targett, T. A., Dunlop, J. S., \& Best, P. N. 2006, MNRAS, 368, 1395

Merloni, A., et al. 2010, ApJ, 708, 137

Natarajan, P. \& Treister, E. 2009, MNRAS, 393, 838

Netzer, H., Lira, P., Trakhtenbrot, B., Shemmer, O., \& Cury, I. 2007, ApJ, 671, 1256
Ouchi, M., et al. 2008, ApJS, 176, 301

Peng, C. Y., Impey, C. D., Rix, H.-W., Kochanek, C. S., Keeton, C. R., Falco, E. E., Lehar, J., \& McLeod, B. A. 2006, ApJ, 649, 616

Peterson, B. M. 2010, in Proceedings of IAU Symposium 267

"Co-Evolution of Central Black Holes and Galaxies", arXiv: 1001.3675

Press, W. H., Teukolsky, S. A., Vetterling, W. T., \& Flannery, B. P. 1992, Numerical Recipes in C: The Art of Scientific Computing (Cambridge: Cambridge Univ. Press)

Reddy, N. A., Steidel, C. C., Erb, D. K., Shapley, A. E., \& Pettini, M. 2006, ApJ, 653, 1004

Riechers, D. A., Walter, F., Carilli, C. L., Bertoldi, F., \& Momjian E. 2008, ApJ, 686, L9

Richards, G. T., et al. 2006, ApJS, 166, 470

Robertson, B., Hernquist, L., Cox, T. J., Di Matteo, T., Hopkins,

P. F., Martini, P., \& Springel, V. 2006, ApJ, 641, 90

Scheuer, P. A. G. 1995, MNRAS, 277, 331

Shankar, F., \& Mathur, S. 2007, ApJ, 660, 1051

Shankar, F., Weinberg, D. H., \& Miralda-Escudé, J. 2009, ApJ, 690,20

Shankar, F., Crocce, M., Miralda-Escudé, J., Fosalba, P., \& Weinberg, D. H. 2010, ApJ, in press, arXiv:0810.4919

Shapiro, S. L. 2005, ApJ, 620, 59

Shen, Y., et al. 2007, AJ, 133, 2222

Shen, Y., Greene, J. E., Strauss, M. A., Richards, G. T., \& Schneider, D. P. 2008, ApJ, 680, 169

Sijacki, D., Springel, V., \& Haehnelt, M. G. 2009, MNRAS, 400, 100

Silk, J. \& Rees, M. J. 1998, A\&A, 331, L1

Small, T. A., \& Blandford, R. D. 1992, MNRAS, 259, 725

Somerville, R. S. 2009, MNRAS, 399, 1988

Springel, V., Di Matteo, T., \& Hernquist, L. 2005, MNRAS, 361, 776

Stanway, E., et al. 2007, MNRAS, 376, 727

Stark, D., Ellis, R. S., Bunker, A., Bundy, K., Targett, T., Benson, A., \& Lacy, M. 2009, ApJ, 697, 1493

Steidel, C. C., Hunt, M. P., Shapley, A. E., Adelberger, K. L., Pettini, M., Dickinson, M., \& Giavalisco, M. 2002, ApJ, 576, 653

Steinhardt, C. L., \& Elvis, M. 2010, MNRAS, in press, arXiv:0912.0734

Tundo, E., Bernardi, M., Hyde, J. B., Sheth, R. K., \& Pizzella, A 2007, ApJ, 663, 53

Ueda, Y., Akiyama, M., Ohta, K., \& Miyaji, T. 2003, ApJ, 598, 886

Urry, C. M., \& Padovani, P. 1995, PASP, 107, 803

Vestergaard, M., Fan, X., Tremonti, C. A., Osmer, P. O., \& Richards, G. T. 2008, ApJ, 674, L1

Vestergaard, M., \& Osmer, P. O. 2009, ApJ, 699, 800

Volonteri, M., \& Rees, M. J., 2005, ApJ, 633, 624

Volonteri, M., \& Rees, M. J., 2006, ApJ, 650, 669

Walter, F., et al. 2003, Nature, 424, 406

Walter, F., et al. 2004, ApJ, 615, L17

Wandel, A., Peterson, B. M., \& Malkan, M. A., 1999, ApJ, 526, 579

Wang, R., et al. 2008, ApJ, 687, 848

Wang, R et al. 2010, ApJ, 714, 699

Willott, C. J., McLure, R. J., \& Jarvis, M. J., 2003, ApJ, 587L, 15

Willott, C. J., et al. 2005, ApJ, 626, 657

Willott, C. J., et al. 2007, AJ, 134, 2435

Willott, C. J., et al. 2009, AJ, 137, 3541

Willott, C. J. et al. 2010, AJ, 139,906

Worseck, G., Fechner, C., Wisotzki, L., \& Dall'Aglio, A. 2007, A\&A, 473,805

Wyithe, J. S. B., \& Loeb, A. 2004, Nature, 427, 815

Woo, J.H., Treu, T., Malkan, M. A. \& Blandford, R. D. 2008 , ApJ, 681, 925

Yoo, J., \& Miralda-Escudé, J. 2004, ApJ, 614L, 25

Yu, Q. \& Tremaine, S. 2002, MNRAS, 335, 965

Yu, Q., \& Lu, Y. 2008, ApJ, 689, 732 

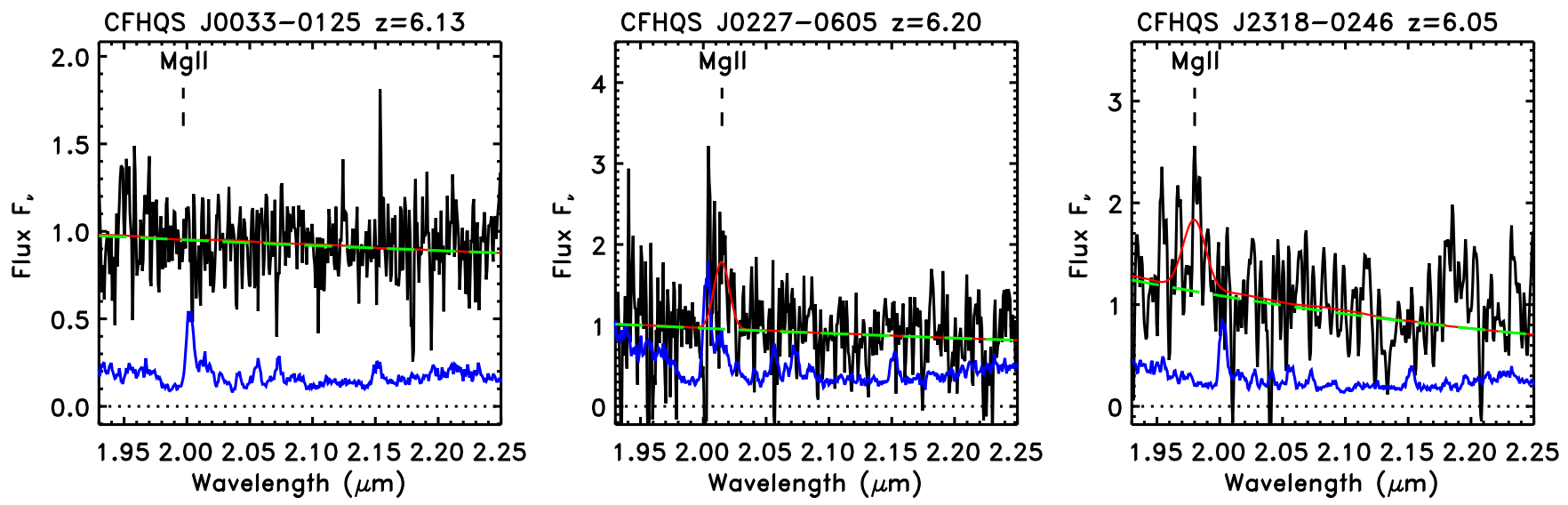

Figure 10. $K$-band spectra of three of the four CFHQS quasars (black line) which did not yield a useful measurement of the Mg II line width. Best-fit model of power law continuum, broadened Fe template and broadened Mg II doublet is shown as a red line. The power law continuum only is shown as a green dashed line. At the bottom of the plot, the blue line is the $1 \sigma$ noise spectrum. The expected location of the Mg II broad emission line is labeled, based on the published Ly $\alpha$ redshift.

\section{APPENDIX}

\section{SPECTRA WHICH DID NOT YIELD USEFUL ESTIMATES ON BLACK HOLE MASSES}

Four CFHQS quasars were observed with the NIRI spectrograph and did not yield measurements of the Mg II emission line width. In all but one case, this was due to insufficient $\mathrm{S} / \mathrm{N}$ for these very faint targets. In the other case it is because the quasar does not have broad emission lines. Therefore black hole masses could not be determined. Here we show the spectra and discuss why Mg II lines could not be measured and what constraints exist on the broad line widths in these quasars. None of these quasars are likely to have broad lines significantly broader than the quasars in Section 3 and therefore the exclusion of these four quasars does not significantly bias the results and analysis presented in this paper.

CFHQS J0033-0125

The optical spectrum of this quasar is very unusual because it shows an extremely weak Ly $\alpha$ line at $z=6.13$ (Willott et al. 2007). Unpublished higher resolution spectroscopy confirms the absence of significant Ly $\alpha$ emission, but does show a sharp continuum break at a Ly $\alpha$ redshift of $z=6.10$ and intervening metal absorption at redshifts up to $z=6.0$ confirming the high-redshift nature of this quasar. The near-IR spectrum has relatively high continuum $\mathrm{S} / \mathrm{N}$ in the range $5-10$ at the expected $\mathrm{Mg}$ II wavelength. As can be seen in Figure 10 there is absolutely no sign of an Mg II line in this quasar, so the lack of Ly $\alpha$ is not just due to dust or IGM absorption, but a lack of broad emission lines such as the quasars investigated by Diamond-Stanic et al. (2009).

\section{CFHQS J0216-0455}

This quasar is the faintest $z \sim 6$ quasar known by some margin with an absolute magnitude $M_{1450}=-22.2$ and could be considered a Seyfert galaxy rather than quasar based on luminosity (Willott et al. 2009). Due to its faintness in the near-IR $\left(K_{\mathrm{AB}} \approx 24\right)$ we did not expect high $\mathrm{S} / \mathrm{N}$ continuum in the NIRI spectrum (which was only composed of 1.75 hours of good weather data). The only reason that we targeted this extremely faint quasar was because the Ly $\alpha$ line is very strong and if the Mg II line were comparably strong, it could have been possible to measure it in $\approx 4$ hours on-source. Neither continuum nor a Mg II line could be seen in the 1.75 hours NIRI spectrum and therefore no spectrum was extracted. We note that the Ly $\alpha$ line is very narrow with FWHM of $1600 \mathrm{~km} \mathrm{~s}^{-1}$ (after correcting for absorption of the blue wing) which implies it has a very high accretion rate, close to the Eddington limit.

\section{CFHQS J0227-0605}

The quasar has $z=6.20$ based on the Ly $\alpha$ line position (Willott et al. 2009). It is quite faint $\left(M_{1450}=-25.03\right)$ and the continuum S/N per pixel in the 2.6 hour long NIRI integration is only $\sim 3$. As shown in Figure 10 the Mg II emission line is detected, but at this redshift, some parts of the line co-incide with the noisiest regions of our spectra due to a combination of a strong sky line and atmospheric absorption. This prevents a reliable measurement of either the line center or the FWHM. The Mg II line appears fairly narrow and the best fit has $z=6.20$ and FWHM $=1900 \mathrm{~km} \mathrm{~s}^{-1}$. However, we do not include it in our analysis because the FWHM is very uncertain.

\section{CFHQS J2318-0246}

This is another very faint quasar $\left(M_{1450}=-24.83\right)$ with a Ly $\alpha$ line at $z=6.05$ (Willott et al. 2009). The 3.9 hours NIRI spectrum shows several peaks around the expected Mg II wavelength (Figure 10). Due to the low S/N it is difficult to constrain the FWHM. The best fit line is at $z=6.07$ and has FWHM $=3100 \mathrm{~km} \mathrm{~s}^{-1}$. Note that the best fit has a very steep, blue continuum which, if extrapolated, is not consistent with the known $z^{\prime}$ and $J$ magnitudes. 